\title{
De huertas y rebaños: reflexiones históricas y ecológicas sobre el papel de la ganadería en al-Ándalus y aportaciones arqueozoológicas para su estudio
}

\author{
Marcos García García y Marta Moreno García
}

PALABRAS CLAVE: Edad Media, andalusí, agricultura, arqueología.

\section{CÓDIGOS JEL: N53, O13, Q15, Q57.}

$E$ l estudio del cambio agrario operado en la Península a raíz de la conquista árabo-islámica del 711 se ha centrado principalmente en el proceso de difusión de la agricultura irrigada. Sin embargo, resulta sorprendente el escaso número de estudios sobre otras prácticas agrarias como las que conciernen al aprovechamiento de las cabañas ganaderas, un tema de investigación que ha sido solo superficialmente explorado debido en parte a suposiciones apriorísticas, como la que presupone la disociación existente entre ganadería y agricultura irrigada en al-Ándalus.

En este trabajo se propone una reconsideración del papel de la ganadería en la economía agraria andalusí. En primer lugar, se exponen una serie de reflexiones que cuestionan la validez de algunos de los argumentos que han contribuido a lastrar el estudio de la actividad pecuaria en al-Ándalus. En segundo lugar, se alude al papel que la práctica ganadera desempeña en el contexto de la producción agraria tradicional, destacando la importancia del estiércol para el mantenimiento de una agricultura intensiva como la irrigada. Por último, se presentan algunos de los principales resultados derivados del estudio arqueozoológico de contextos andalusíes, cuya lectura agroecológica contribuye a mejorar nuestra comprensión del sistema agrario andalusi y del papel que pudo desempeñar en él la actividad pecuaria. 


\title{
On orchards and flocks: historical and ecological reflections regarding the role of animal husbandry in al-Ándalus using archaeozoological data
}

\author{
KEYWORDS: Middle Ages, Andalusi, agriculture, archaeology.
}

\author{
JEL CODES: N53, O13, Q15, Q57.
}

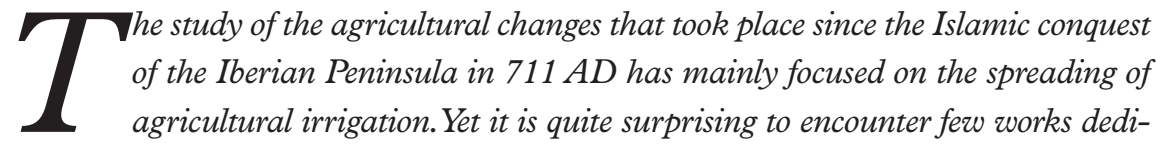
cated to other agricultural practices that were equally relevant within the productive context of any preindustrial economy, such as animal husbandry. Research on the main practices of husbandry and its management have received little attention due in part to virtually unfounded a priori assumptions, such as the existence of dissociation and competition between this farming subsector and irrigation agriculture.

The following study aims to reexamine evidence on the role of domestic animal farming and management in the Andalusi agricultural economy. First, a critical analysis is presented of the causes of the sparse attention paid to the study of the animal economy in al-Ándalus. Second, the paramount importance of animal husbandry within premechanized farming contexts is shown along with the crucial role of a by-product such as animal manure for the maintenance of fertility in irrigated agricultural land. Lastly, we offer an agroecological interpretation of results obtained from the analysis of assemblages of animal remains from Andalusi archaeological sites, aiming to offer a glimpse into the agricultural system of al-Ándalus and the role played by animal husbandry within it.

Recepción: 2017-04-26 - Revisión: 2017-11-20 - Aceptación: 2017-11-30

Marcos García García [orcid.org/0000-0002-2080-5098] es contratado de investigación FPU en el Departamento de Historia Medieval y CC.TT.HH. de la Universidad de Granada. Dirección para correspondencia: cl Granada, 42c 1. ${ }^{\circ}$ izq. HuétorVega, 18198 Granada (España).E-mail:marcosgarcia@ugr.es

Marta Moreno García [orcid.org/0000-0002-6735-9355] es científica titular en el Instituto de Historia del Centro de Ciencias Humanas y Sociales del CSIC (G.I. Arqueobiología). Dirección para correspondencia: Instituto de Historia (CCHS-CSIC), c/Albasanz, 26-28, 28037 Madrid (España). E-mail: marta. moreno@cchs.csic.es 
"[...] el regar destruye y disipa mucho la tierra, que se lleva la flor della, y deja solamente el arena; $y$ por esto la tierra que se riega ha menester mucho estercolarse»

G. Alonso de Herrera (1513). Agricultura General, t. III, lib. IV, cap. IV.

\section{INTRODUCCIÓN}

La investigación histórica y arqueológica sobre al-Ándalus ha dedicado gran atención al estudio del mundo rural y al análisis de la formación de los paisajes agrarios. Sin embargo, en el tratamiento historiográfico sobre estas cuestiones se detecta un enfoque que prioriza el examen de un limitado número de estrategias agrarias (esencialmente, la agricultura irrigada), al tiempo que relega al desempeño de un papel menor y marginal otras opciones productivas. De resultas, en ocasiones se han realizado interpretaciones que parten de esquemas excesivamente rígidos sobre el funcionamiento de los agroecosistemas históricos, en detrimento de una consideración orgánica y ecológica del conjunto de motivaciones e intereses que determinan los procesos de trabajo campesino, que son, al fin y al cabo, el eje en torno al cual se articula cualquier sistema agrario.

Nuestra intención queda lejos de cuestionar la relevancia del papel de la agricultura irrigada en la organización de la economía rural islámica y, por extensión, andalusí. No es baladí la consideración de los espacios agrícolas irrigados como la máxima expresión agropecuaria de la sociedad andalusí (Eiroa, 2012:392), como tampoco lo es el hecho de que los principios generales que rigen la tecnología hidráulica que los dota de sentido (formulados y sistematizados por Miquel Barceló y su equipo a partir de la década de los ochenta del siglo pasado), hayan dado cuerpo a una línea de investigación de una enorme capacidad explicativa más que demostrada, como es la arqueología hidráulica (Barceló, 1989, 1995; Kirchner \& Navarro, 1994). Consideramos, asimismo, de extraordinaria relevancia la dimensión social de los mecanismos sobre los que se basa este tipo de agricultura, por lo que creemos que andaba en lo cierto M. Barceló (1989: xv) cuando señaló que el espacio irrigado es el resultado de una decisión social que produce formas específicas del proceso de trabajo campesino y que impone condiciones específicas de organización social. No resulta pertinente ahondar aquí en estas consideraciones que, por otro lado, son de sobra conocidas. Sobre lo que sí nos interesa llamar la atención es sobre el hecho de que, como han puesto de manifiesto diferentes autores (entre ellos el propio Barceló [1988]) la agricultura de regadío, aun siendo muy importante, no debe considerarse como la única línea económica existente en al-Ándalus (Malpica, 2011, 2012; Retamero, 2002, 2011; Torres, 1988). 
En este sentido, cabe señalar que la ganadería ${ }^{1}$ no ha sido, salvo en contadas ocasiones a las que se aludirá, adecuadamente integrada en el estudio del sistema agrario andalusi ${ }^{2}$. El resultado, tal y como veremos, ha supuesto el desplazamiento de la práctica pecuaria al desempeño de un papel marginal en el marco de las estrategias productivas desarrolladas por las comunidades rurales en al-Ándalus, a pesar de tratarse de una actividad agraria que tradicionalmente ha tenido una enorme relevancia en la conformación de la economía mediterránea preindustrial (Braudel, 1953; Halstead, 1987, 1993; Horden \& Purcell, 2000). Como acertadamente recuerda V. Martínez Enamorado (2003: 103; 2009: 75), todas aquellas estrategias tendentes a resolver problemas subsistenciales básicos, que no son más que la puesta en funcionamiento de soluciones a problemas alimentarios, se resumen bajo los conceptos de agricultura y ganadería, al mismo tiempo y sin distinción, por lo que ambas actividades no pueden desvincularse en el proceso productivo y deben integrarse forzosamente en un mismo paquete de análisis y estudio.

En este trabajo nos proponemos abordar un tema complejo como es el de la relación que pudo establecerse entre la actividad ganadera y agrícola en al-Ándalus ${ }^{3}$. Para ello, consideramos esencial el recurso a fuentes históricas alternativas a las escritas, que no complementarias (Barceló, 1992: 458; Barceló et al., 1988: 11-12), habida cuenta de que, justamente, la invisibilidad documental de la ganadería andalusí podría haber fundado su escaso éxito historiográfico (Cara, 2009: 172) y, en consecuencia, la consideración ancilar de esta actividad con respecto a la agricultura irrigada en el conjunto de las opciones productivas agrarias. Una de las líneas de análisis arqueológico de mayor potencialidad

1. Entendiéndola, en un sentido amplio, como el conjunto de prácticas adoptadas por los grupos humanos para la explotación de un "selecto" grupo de animales.

2. Aunque en este trabajo la atención se centra exclusivamente sobre la actividad pecuaria, no quisiéramos dejar pasar la oportunidad de poner de relieve la necesidad de incorporar plenamente en la investigación sobre los procesos de trabajo campesino en al-Ándalus y las estrategias dirigidas a asegurar la reproducción social, el estudio de otras modalidades de explotación del medio, como la agricultura de secano (RETAMERO, 2011), el uso del bosque, de los marjales y humedales (GUTIÉRREZ LLORET, 1995; MALPICA 2017), la caza y pesca o el aprovechamiento de los recursos salineros (GARCÍA-CONTRERAS, 2011; MALPICA, 2008), entre otras.

3. Hacemos nuestras las palabras de A. MALPICA (2000: 249) cuando advierte de que considerar la historia de al-Ándalus como un todo unitario y sin transformaciones supone negar lo que significa la misma Historia. Resulta, por ello, necesario apuntar que a lo largo de sus casi ocho siglos de existencia, las estructuras agrarias andalusíes no permanecieron estáticas. Se ha señalado la importancia de dos factores decisivos que, en su desarrollo, debieron forzosamente influir sobre la capacidad y orientación de las opciones productivas de los grupos campesinos andalusíes, como son la presión del Estado y su demanda de tributos, y la influencia de las redes comerciales dirigidas principalmente al abastecimiento urbano. Así pues, la misma dinámica histórica, unida al mosaico geográfico y ecológico que conforman las diferentes microrregiones ibéricas, obligan a proceder con cautela a la hora de formular patrones generales para definir las múltiples y variadas estrategias de explotación del medio y, particularmente, de gestión y aprovechamiento ganadero. 
informativa sobre esta cuestión es la arqueozoología (el estudio de los restos de animales recuperados en contexto arqueológico), ya que permite arrojar luz sobre los sistemas de manejo y aprovechamiento de las cabañas ganaderas y, de esta forma, generar conocimiento histórico de interés sobre aspectos esenciales del sector pecuario ignotos a través de la documentación escrita (e.g. Audoin-Rouzeau, 1993; Morales, 1996; Moreno, 2013a; O’Connor, 2014; Salvadori, 2015).

Así pues, el objetivo que nos marcamos pasa fundamentalmente por ofrecer una serie de elementos de reflexión que, desde un enfoque analítico que privilegie la consideración de los factores sociales y ecológicos en el funcionamiento de los sistemas agrarios tradicionales, y a la luz de la evidencia documental y arqueozoológica disponible, sirvan para articular un argumento esencial en este trabajo, cual es que -anticipando la principal conclusión-, lejos de ser dos actividades disociadas como se ha venido planteando, la ganadería y la agricultura irrigada debieron establecer una relación simbiótica sin la cual no hubiera sido posible la intensificación agrícola que supuso la implantación y difusión del regadío en al-Ándalus. Con este propósito, trataremos en primer lugar de esbozar un balance somero del tratamiento historiográfico que se ha dado al estudio de la ganadería en al-Ándalus, poniendo el acento sobre algunos de los elementos que, a nuestro juicio, permiten caracterizar los rasgos esenciales de esta línea de investigación histórica. Centraremos la atención, en segundo lugar, sobre el papel que desempeña la ganadería en el marco de la producción agraria tradicional en el Mediterráneo, señalando la relevancia de esta actividad como opción social ante el riesgo productivo, así como el carácter ineludible que tiene el uso de un producto secundario de origen animal como es el estiércol para el mantenimiento de las prácticas agrícolas intensivas. Por último, presentaremos algunos de los resultados principales derivados del estudio del registro arqueozoológico que, una vez integrados con lo que sabemos a partir de la información documental, nos ayuden a mejorar nuestro conocimiento sobre la economía animal y su papel en el complejo agrario de al-Ándalus.

\section{EL ESTUDIO DE LA GANADERÍA EN AL-ÁNDALUS: UN BALANCE SINTÉTICO}

La escasa importancia atribuida a la actividad pecuaria en al-Ándalus es, al decir de L. Cara (2002: 456), un lugar común dentro de la historiografía medieval española ${ }^{4}$. No obs-

4. Buena prueba de ello es la ausencia de este tema en algunos de los principales trabajos colectivos de síntesis sobre ganadería de época medieval en la Península (e.g., GERBET, 2002; Gómez PANTOJA, 2001a; MuÑoz \& DíAz LóPEZ, 2002). 
tante, contamos actualmente con una serie de trabajos que han tratado de integrar la actividad ganadera en el estudio del mundo rural andalusí, focalizado tradicionalmente de manera prioritaria sobre el sector estrictamente agrícola. Para los objetivos del presente trabajo resulta necesario, por lo tanto, apuntar una serie de ideas generales que consideramos de importancia en relación con esta línea de investigación que nos permitan perfilar una caracterización a grandes rasgos del tratamiento historiográfico que ha recibido el estudio de la ganadería andalusí 5 .

En primer lugar, resulta esencial apuntar que el conocimiento que poseemos sobre el sector ganadero en al-Ándalus se deriva en su mayor parte de las referencias a esta actividad contenidas en las fuentes escritas ${ }^{6}$. Así, no sorprende que, debido a la relativa abundancia de documentación escrita generada durante el periodo nazarí y el periodo inmediatamente posterior a la conquista castellana, sea la ganadería del antiguo reino de Granada la mejor conocida de al-Ándalus. Coincidimos, por lo tanto, una vez más con L. Cara (2009: 171) cuando atribuye a la escasa presencia de menciones al sector pecuario en la documentación escrita un carácter esencial a la hora de explicar los motivos por los cuales el balance de la investigación sobre la ganadería en al-Ándalus resulta, a su juicio, descorazonador ${ }^{7}$.

5. Nuestro propósito en esta sección no pasa por ofrecer un balance crítico detallado del conocimiento histórico que poseemos actualmente acerca de la ganadería andalusí por considerar esta una tarea que supera los objetivos del presente trabajo, si bien a lo largo de él se hará referencia a algunos de los elementos de mayor significación que se han propuesto para definir las líneas fundamentales que caracterizaron a esta forma de explotación agraria en al-Ándalus. La producción científica sobre esta temática es, en realidad, bastante fragmentaria, con acusadas disparidades regionales y cronológicas, y con una evidente ausencia de planteamientos generales y análisis de síntesis. En nuestra opinión, los principales estudios que han tratado de manera parcial o monográfica este tema de investigación se los debemos a CARA (1988, 1996, 2002, 2004, 2009), CARA y RODRÍGUEZ (1989a, 1989b), GLICK (1970: 22-30), LAGARDĖRE (1993: 441-479), MALPICA (2011, 2012), MARTín (2007: 317-341), Martínez Enamorado (2003: 103-139), Ramos (1988), Trillo (1998, 1999, 2004, 2011) y WATSON (Inédito, 2007). Será principalmente de estos trabajos de los que extraigamos algunos de los argumentos a los que se aludirá en las reflexiones que siguen.

6. Dado que, como señala ToRREs (1988: 136), la información existente a este respecto en la documentación escrita es escasa, el recurso a otro tipo de evidencias resulta imprescindible. Como se aludirá más adelante, la contribución de la arqueología al conocimiento del papel que jugó la ganadería en al-Ándalus no resulta una novedad (e.g., CARA \& RODRÍGUEZ, 1989a, 1989b; TORRES, 1988), si bien es cierto que este tipo de estudios, principalmente desde los planteamientos de la arqueología del paisaje, han recibido recientemente nuevos impulsos (GARCÍA-CONTRERAS, MALPICA \& VILLAR, 2013; MALPICA et al., en prensa; VILLAR \& GARCÍA GARCÍA, 2017). Con todo, ya hemos señalado que consideramos la arqueozoología como la disciplina de análisis arqueológico de mayor potencial para conocer los sistemas de explotación y gestión de los animales en el pasado, por lo que le dedicaremos especial atención a lo largo de la tercera sección del presente trabajo.

7. La invisibilidad de la ganadería andalusí en la tradición literaria islámica (ÁLVAREZ DE MORALES, 1990) se hace incluso más evidente cuando se compara con la rica documentación disponible 
En segundo lugar, una de las principales conclusiones obtenidas a partir del estudio de la información documental disponible, y en la que convergen muchos de los trabajos dedicados al análisis de los sistemas agrarios en al-Ándalus, es la escasa relevancia de la ganadería durante este periodo ${ }^{8}$. Desde nuestro punto de vista, y como nos esforzaremos en demostrar a lo largo de esta contribución, consideramos que este resultado es, en gran medida, consecuencia directa de la naturaleza de las fuentes empleadas para su estudio y reflejo, al mismo tiempo, de los límites informativos de la documentación escrita. Para el caso concreto de la actividad ganadera, es necesario tener presente que, por su propia naturaleza, esta ha representado tradicionalmente un fenómeno altamente esquivo al registro documental (Cara, 1996; Halstead, 1996), salvo en los casos en los que conforma sistemas económicos altamente especializados y de gran escala productiva, como fue, por ejemplo, el de la ganadería mesteña (Klein, 1920; García Martín, 2011). Este rasgo no es único, además, del mundo andalusí, sino una característica compartida con otras tradiciones literarias como la del mundo clásico grecorromano, en la que los temas estrictamente agrícolas tienen una visibilidad mucho mayor que los ganaderos (Hodkinson, 1988; Kron, 2008). Como apunta J. Gómez Pantoja (2001b), la pastoría es una realidad excepcionalmente opaca a la documentación escrita, por lo que resulta difícil encontrar en las fuentes escritas lo que raramente aparece en ellas.

De mayor interés para nuestros objetivos actuales resulta un tercer elemento común que encontramos en gran parte de los trabajos de investigación que se han dedicado al análisis de la ganadería andalusí como es el de la supuesta contradicción, aparentemente existente, entre las prácticas ganaderas y la agricultura de regadío. Ya que la perspectiva de nuestro trabajo está enfocada esencialmente a examinar, desde una óptica agroeco-

para otros territorios medievales hispánicos, como los reinos de Jaén y Córdoba (ARGENTE DEL CASTillo, 1991), Navarra (Díaz de Durana \& Fernández de LAERra, 2002), Castilla (PAscuA, 2007) o Aragón (PAscua, 2011).

8. No por resultar una obviedad consideramos prescindible precisar que la opción ganadera no puede ser considerada de manera unitaria para todo el conjunto de al-Ándalus, pues existen, lógicamente, diferencias evidentes en el peso atribuido por parte de la historiografía a esta actividad productiva en función del ámbito espacial y el contexto temporal en cada caso. Así, por ejemplo, en dos zonas inmediatamente próximas en términos geográficos como son la Vega de Granada y el Quempe (situado al sur de la capital), la importancia asignada a esta actividad difiere significativamente: mientras que para la primera se ha señalado que, durante época nazarí, la ganadería representaría un recurso complementario de no demasiada trascendencia (OCAÑA, 1974: 468), para la segunda, el estudio realizado por VILLAR (2016: 984) le permite concluir que el peso de la ganadería debió ser muy significativo. Junto a diferencias espaciales las hay también temporales. Por ejemplo, MARTínez EnAMORADO (2003: 137) considera que el desarrollo de mecanismos de comercialización de productos ganaderos en la comarca malagueña de la Algarbía (inferido por la aparición de unas pocas familias propietarias de grandes rebaños) solo se produce en el periodo tardonazari, siendo impensable para los periodos anteriores, en los que no hay espacio para una ganadería competitiva y comercial a gran escala. 
lógica, la integración entre agricultura y ganadería en al-Ándalus, consideramos importante dedicar atención a esta cuestión, ofreciendo asimismo una serie de argumentos que pongan en evidencia la inconsistencia que, a nuestro juicio, presenta la noción de la disociación entre ambas actividades en el marco productivo de una economía agraria preindustrial como fue la andalusí.

$\mathrm{El}$ argumento de fondo puede resumirse en dos incompatibilidades de tipo técnico que presenta la actividad ganadera en un sistema de organización agrícola en el que la irrigación es el factor determinante de la economía rural, tal y como se ha puesto de manifiesto para gran parte de al-Ándalus. De un lado, la fragilidad de los elementos técnicos del sistema (canales, surcos, caballones, etc.), fácilmente destruibles por el paso y la acción del ganado; de otro, el carácter permanente de la producción en la que no hay período de descanso en la tierra cultivada durante el cual el ganado pueda acceder a los espacios agrícolas para pastar en ellos.

Hasta donde nosotros sabemos, el primer autor que formuló la noción de un sistema agrario en al-Ándalus definido por la disociación entre agricultura y ganadería fue A. M. Watson (inédito, 2007) ${ }^{9}$. En esencia, su razonamiento pasaba por asumir que el panorama agrario que se describe en los famosos libros de agricultura andalusíes refleja una realidad en la que, como consecuencia de la no difusión al mundo islámico del sistema de open-fields característico de la Europa medieval cristiana (en el cual agricultura, de secano, y ganadería se encontraban estrechamente vinculadas entre sí), la agricultura en al-Ándalus estuvo en riesgo constante debido a la falta de integración con la ganadería ${ }^{10}$. En sus líneas generales, esta conclusión supone una continuación de algunas de las ideas expuestas en la "tríada» de sus primeros y más relevantes trabajos sobre el mundo rural islámico $(1974,1981,1998)$, fundamentalmente de la que sostiene que la «revolución» que supuso la expansión árabe medieval para los sistemas agrícolas tradicionales mediterráneos permitió el abandono del barbecho, favoreciendo de esta forma una rotación continua de los cultivos en los terrenos bien irrigados y la reducción al mínimo de las tierras de secano potencialmente utilizables como fuente de alimentación del ganado.

9. Aunque ya en su obra seminal publicada por primera vez en 1983 (WATSON, 1998) abordaba tangencialmente esta cuestión, los postulados de este investigador acerca de la relación entre agricultura y ganadería en al-Ándalus quedaron plenamente formulados en su contribución al congreso homenaje a P. Guichard celebrado en 1996 entre Granada y Valencia (WATson, inédito). Debido a que las actas de aquel congreso nunca llegaron a prensa, el trabajo de Watson fue finalmente publicado, con pequeñas adiciones y modificaciones, en 2007 en las actas de la XXXVIII Settimana di Studi della Fondazione Istituto Internazionale di Storia Economica "F. Datini" (Prato, Italia).

10. The agriculture of Muslim Spain was at risk because of the lack of integration of crop and animal production [...]. Instead of these two activities being joined together [...], each depending on and reinforcing the other, they were hostile to one another (WATSON, 2007: 262). 
Nuestra intención no pasa aquí por formalizar una crítica de raíz a los postulados presentados por Watson en aquellos dos trabajos (inédito; 2007), más aun cuando consideramos que suponen un avance muy significativo en el conocimiento del mundo islámico clásico en el que introdujo, además, elementos de enorme interés para el estudio de los sistemas agrarios medievales, como son la cuestión de la transferencia de conocimientos geopónicos entre sociedades culturalmente diferenciadas o la singularidad europea del sistema de campos abiertos. El motivo esencial para traer a colación las apreciaciones de Watson acerca de la relación entre agricultura y ganadería en al-Ándalus es la repercusión que estas tuvieron en la investigación histórica sobre el sistema agrario andalusí, pues fueron recogidas y ampliamente aceptadas por otros investigadores que, en estudios posteriores, dieron por buena la disociación entre ambas actividades planteada por el autor canadiense. Desde nuestro punto de vista, este hecho resulta de notable interés, ya que la principal fuente informativa de la que se sirvió para postular la separación entre estos dos subsectores agrarios (los kutub al-filāha, los famosos libros de agricultura andalusíes) presenta serios problemas que resulta difícil ignorar. Tal y como puso de manifiesto F. Retamero (1998), los conocimientos agrarios codificados en los tratados de geoponimia andalusíes no responden a lo que ocurre en los territorios campesinos alejados de los ahrwāz de las mudün (los alfoces de las ciudades), porque el objetivo manifiesto de dichos textos es fomentar una práctica agrícola «de máximos» dirigida a la obtención de los rendimientos más elevados. El mismo Watson reconoce que their instructions seem to be aimed at estates or communities concentrating on the production of fruits, vegetables, legumes, flowers and industrial crops, often on irrigated land (2007: 249). Por lo tanto, creemos que existen serias dudas a la hora de otorgar validez a la realidad que se refleja en este tipo concreto de producción literaria (vinculada evidentemente al ámbito del poder) en relación con la forma en que se articulan los dos componentes elementales del sistema agrario puesto en marcha por las comunidades de productores rurales.

En nuestra opinión, y como trataremos de demostrar, el argumento que sostiene la supuesta disociación entre agricultura irrigada y ganadería en al-Ándalus se invalida por tres motivos fundamentales. En primer lugar, se deriva en su mayor parte del análisis de una fuente histórica que presenta serios problemas -a los que nos hemos referido brevementerealizado, además, de manera parcial, ya que existen diversas evidencias documentales, incluso en los mismos tratados de agricultura, que permiten postular un mayor nivel de integración entre ambas actividades. En segundo lugar, surge de una deficiente comprensión de la potencialidad funcional que las características ecológicas de las regiones mediterráneas ofrecen para la puesta en práctica de mecanismos de intensificación y diversificación productiva que, lejos de promover la disociación entre actividad agrícola y ganadera, favorecen su integración simbiótica sin que deban entrar necesariamente en conflicto.Y, por último, y como apunta L. Cara (2002: 457; 2009: 174), ignora el papel 
de la ganadería como elemento imprescindible para la agricultura, presuponiendo que la intensificación productiva agraria durante época islámica fue posible solo mediante la irrigación, lo que soslaya el problema del estiércol. A lo largo de la siguiente sección trataremos de dar forma a estos argumentos.

\section{LA GANADERÍA EN EL MARCO DE LA PRODUCCIÓN AGRARIA ANDALUSÍ}

Con el fin de replantear el problema de la relación entre agricultura y ganadería en alÁndalus, dedicaremos este apartado a aportar una serie de reflexiones que sitúen en sus justos términos el papel que, creemos, pudo desempeñar la explotación de las cabañas ganaderas en el marco de las estrategias productivas desarrolladas por las comunidades rurales andalusíes. Para ello, se hace preciso en primer lugar considerar el contexto sociopolítico en el que se inscribe el sistema de producción agrario que, tal y como se ha venido caracterizando, estaría definido principalmente por el elevado nivel de autonomía de las comunidades campesinas en lo que concierne a la selección de sus opciones y estrategias productivas. A continuación, mediante el recurso a la noción de riesgo productivo y la alusión a las estrategias básicas de su disipación (diversificación e intensificación), trataremos de integrar en el modelo del complejo agrario andalusí el componente ganadero a través de dos enfoques que se imbricarán. De un lado, una lectura alternativa de la información documental disponible que, en nuestra opinión, refleja un panorama agrario en el que agricultura y ganadería aparecen ciertamente integradas. De otro lado, una serie de reflexiones derivadas de las observaciones etnográficas acerca del funcionamiento de los sistemas agrarios tradicionales en el Mediterráneo.

Una cuestión que ha suscitado cierta controversia en el seno de la investigación histórica sobre el período andalusí ha sido el grado de autonomía de las comunidades rurales en lo referente a la ordenación de sus procesos de trabajo. Dado que la idea de un mundo campesino "cerrado en sí mismo» cuyas relaciones con el Estado se limitan al pago periódico del impuesto coránico se ha revelado rígida e insuficiente (Eiroa, 2012; Malpica, 2009; Manzano, 2012), resulta imprescindible mejorar nuestra comprensión acerca de la relación establecida entre las comunidades campesinas y los poderes externos a ellas (Barceló, 1992). En otras palabras, sigue siendo necesario intensificar el estudio de los procesos de trabajo campesino y del mayor o menor grado de su dominación desde el exterior, ya que solo a partir del (re)conocimiento de las estrategias productivas adoptadas por los grupos productores en al-Ándalus resulta posible ponderar el grado de penetración de poderes externos (públicos o no) en estas comunidades. Así pues, haremos uso de la noción, en la línea trazada por M. Barceló, de gestión local y autónoma de los pro- 
cesos de trabajo en el marco social, político y económico que conforman las qurā o alquerías, base desde la cual se organizaron, al menos durante sus primeros tiempos, las estrategias productivas de los grupos campesinos andalusíes, sin asumir con ello una caracterización de un mundo campesino autosuficiente que se produce y reproduce de espaldas al poder central.

Hecha esta apreciación, consideramos necesario destacar la importancia capital que el concepto de riesgo productivo y las diferentes formas de gestionarlo presentan en un contexto sociopolítico en el que la toma de decisiones productivas es gestionada de manera autónoma y a nivel local por las comunidades rurales y en un marco geográfico como el Mediterráneo (Glick, 2004; Halstead \& O’Shea, 1989; Horden \& Purcell, 2000). Disponemos de abundante evidencia etnográfica e histórica que permite reconocer que, en las regiones mediterráneas, la estrategia básica de supervivencia ha consistido tradicionalmente en la disipación del riesgo productivo por medio de la explotación del mayor número de nichos ecológicos posible, la utilización de la mayor cantidad de recursos disponibles y el diseño de estrategias tendentes a aumentar la producción por unidad de explotación. Diversificación e intensificación son, pues, los dos puntales básicos sobre los que descansan los sistemas agrarios tradicionales en el Mediterráneo (Halstead, 2014; Horden \& Purcell, 2000; Marston, 2011).

En este sentido, cabe señalar que la explotación ganadera permite la reducción del riesgo productivo, tanto mediante la diversificación como la intensificación de la producción agraria, lo que la convierte, como ya se ha dicho, en uno de los elementos clave en la conformación de cualquier economía preindustrial. Debido tanto al carácter multifuncional de su producción como a la flexibilidad de las estrategias de gestión de sus recursos, la opción ganadera ha contribuido tradicionalmente a aumentar el grado de $d i$ versificación de la producción agraria en tres sentidos. En primer lugar, mediante la explotación de biotopos como bosques, zonas montañosas y riscosas o marjales y humedales que, de otro modo, ofrecen escasos volúmenes de nutrientes aprovechables de manera directa por los grupos humanos (Forbes, 2000). En segundo lugar, permitiendo el acceso a una mayor variedad de recursos explotados gracias al aprovechamiento de productos tanto recurrentes (leche, lana, estiércol, fuerza de tracción...) como no recurrentes (carne, grasa, tendones, pieles, huesos...) (Sherratt, 1981). Por último, a través de la utilización de los animales como vehículos para el almacenamiento indirecto de excedentes agrarios y su uso como capital animal que puede ser consumido en caso de necesidad (Flannery, 1969; Halstead, 1992, 1993).

En el caso concreto de las regiones mediterráneas, se ha puesto de manifiesto que la oposición estereotipada entre ager y saltus, que en numerosas ocasiones ha derivado en 
la noción de la exclusión mutua entre agricultura y ganadería (Wickham, 1983), se invalida por la naturaleza fragmentada de las múltiples microrregiones que la componen (Horden \& Purcell, 2000). La alternancia de zonas con suelos cultivables y áreas imposibles de explotar agrícolamente, usualmente calificadas como marginales ${ }^{11}$, característica fundamental de los paisajes mediterráneos, hace posible la explotación de ganado doméstico sin que la gestión de los recursos necesarios para su mantenimiento tenga que entrar forzosamente en competición con los destinados a la alimentación humana (Forbes, 1998; Montserrat \& Fillat, 1990). La opción productiva ganadera permite, de esta forma, la explotación de extensos espacios de terreno de gran presencia en las regiones mediterráneas a través de la ingestión, por parte de los animales, de la biomasa que contienen y su conversión en productos ganaderos.

Volviendo de nuevo la vista a al-Ándalus, resulta difícil negar que el regadío, entendido tal y como se ha venido haciendo como la estrategia básica, que no única, de producción desarrollada por las comunidades campesinas andalusíes, impone unas rígidas condiciones sobre la organización del espacio agrario. Es sabido que este tipo de agricultura requiere grandes inputs de trabajo por unidad de superficie, lo que permite la explotación de unos espacios agrícolas altamente productivos. De resultas, las parcelas de tierra así cultivadas en contextos tradicionales o preindustriales no suelen alcanzar unas dimensiones excesivamente amplias y tienden a localizarse a escasa distancia de las áreas de residencia, condiciones ambas necesarias para el buen desarrollo de las prácticas intensivas (escarda, labranza, rotaciones, abonado, etc.) asociadas a este tipo de agricultura (Halstead, 2014; Jones, 2005; Trillo, 2004). Así pues, aun contando con la existencia de una enorme variabilidad para ambos parámetros (tamaño de los espacios irrigados y proximidad a las zonas de habitación), no parece arriesgado sugerir que el espacio que no estuvo explotado de manera directa, continua e intensiva en al-Ándalus tuvo que ser, necesariamente, extenso ${ }^{12}$.

Lógicamente, la existencia de tierras potencialmente aprovechables por la ganadería no implica su uso como tal, que debiera en todo caso explicarse como resultado de la suma de decisiones colectivas por parte de las comunidades campesinas que son las que cons-

11. Nos referimos a biotopos estériles desde un punto de vista estrictamente agrícola, pero que pueden ser explotados de otras formas no solo ganaderas, sino también a través de la recolección, la caza, etc.

12. Nos parece, a este respecto, un sobredimensionamiento desmesurado de la extensión del regadío la apreciación que hace WATSON (1983/1998: 235) cuando señala que, en el mundo islámico, los campos sin cultivar y los bosques se mantenían al mínimo. Posiblemente no entendamos bien qué quiere decir con esta frase o se trate de un problema de traducción del original en inglés (al que no hemos podido acceder) al castellano. 
tituyen, en última instancia, las estrategias agrarias. Dicho lo cual, y siguiendo nuestra línea argumental, es importante considerar igualmente la situación jurídica atribuida a los espacios incultos en el marco del funcionamiento de las clases de tierras en una sociedad islámica medieval como fue la andalusí, ya que sabemos que, en el mundo rural, toda zona urbanizada ('umrān) tenía a su disposición un terreno comunal (harim baladī) inalienable a dicha población, cuyo uso principal estaba destinado a servir de zona de herbaje para el ganado local (Camarero \& Cano, 2011; Lagardère, 1993: 459; 1996; Trillo, 2004: 80). Este aprovechamiento colectivo de los terrenos baldíos locales con fines ganaderos estaba, además, favorecido por la comunidad de pastos existente al menos en determinadas regiones durante época nazarí (Ramos, 1988) y amparada en un conocido hadìt del Profeta (Linant de Bellefonds, 1959). Creemos, por lo tanto, que estos argumentos deben tenerse en cuenta a la hora de plantear, como en ocasiones se ha hecho, que la presencia de campos de cultivo irrigados supone per se un factor limitante para el desarrollo de la práctica ganadera, ya que, como hemos tratado de poner de manifiesto, y siguiendo la opinión de A. Malpica (2011), supone un error de consideración al desestimar el excedente de tierras potencialmente disponibles para el mantenimiento de las cabañas ganaderas.

Por otro lado, creemos de gran importancia señalar que, aunque menos estudiada, la agricultura de secano formó igualmente parte, junto a la de regadío, del diseño de las estrategias de producción y reproducción social de los grupos campesinos andalusíes, lo que permitió una diversificación que incluía la posibilidad de articular agricultura y ganadería (Malpica, 2011; Retamero, 2011; Trillo, 2011). De hecho, a partir de una serie de menciones contenidas en las fuentes escritas (algunas de ellas utilizadas por Watson), diversos autores han puesto sobre la mesa una serie de argumentos que contribuyen a cuestionar la disociación taxativa entre ambas actividades, particularmente en el marco de la agricultura de secano que, evidentemente, también existió en al-Ándalus ${ }^{13}$.

En su reseña al trabajo de Watson (1998), F. Aubaile-Sallenave (1984) expone una serie de críticas a algunas de las interpretaciones que este investigador realiza sobre la realidad descrita en los textos que utiliza. La más relevante para nuestro propósito actual es

13. En relación a este aspecto, BolENs (1994: 171) recomienda matizar la clásica división entre agricultura de secano y de regadío, siendo más plausible la intercalación en el paisaje de islotes de cultivo intensivo irrigado en zona de secano, tierras de alfalfa que son polos de atracción para los rebaños de montaña en altos valles cultivados en huertas. La misma autora, siguiendo a HALPERIN (1956) señala que el regadío es muy restringido y el secano demasiado pobre para que puedan constituirse en mundos separados. Nuestros agrónomos andaluces [sic] pasan insensiblemente del uno al otro, sin hacer de ellos categorías de cultivo claramente separadas. WATSON (1998: 263) menciona igualmente esta idea cuando señala que no existía una separación marcada entre terrenos de regadio y de secano. 
la que la autora francesa vierte sobre la supuesta supresión del barbecho en el mundo islámico, una práctica que, al contrario de lo que opina Watson, sí aparece mencionada y recomendada en la tratadística geopónica andalusí, como también constata L. Bolens (1994); por lo que, cuando Watson nos dice que en la mayoría de los manuales no se recomienda el barbecho y ni siquiera se habla de él (1998: 258), lo que hace es expresar su opinión personal y no la de los agrónomos medievales (Aubaile-Sallenave, 1984: 254).

Efectivamente, en los mismos textos utilizados por Watson se recomienda el uso del barbecho ${ }^{14}$, una práctica agraria que genera un margen de integración entre ganadería y agricultura en el que ambas actividades se ven mutuamente beneficiadas: la una mediante el aprovechamiento de una fuente de alimentación (el rastrojo) y la otra gracias al efecto fertilizador que tienen las deyecciones depositadas en el terreno agrícola. Mencionaremos únicamente unos cuantos ejemplos. En su tratado de agricultura, Ibn al'Awwām (siglos XII-XIII) hace mención expresa a la práctica del redileo, señalando que, tras la cosecha, es recomendable llevar a la cabaña ovina, caprina y bovina al campo segado dejado en rastrojo, especificándose que los tallos de las plantas deben cortarse a media altura con la clara intención, creemos, de servir de alimento para el ganado. Ibn Luyun (siglo XIV) recomienda igualmente la entrada de ganado menor (ovino, en este caso) en terrenos agrícolas con el objeto de estercolar la tierra, para lo cual deben seguirse ciertas normas que eviten la sobrefertilización de los campos (permanencia máxima de dos o tres noches) y la germinación de las semillas contenidas en los excrementos (labrando el terreno antes de cinco meses). Por su parte, Ibn Bașsâal (siglo XI) señala la necesidad de estercolar la tierra, indicando que esta práctica es solo realizable en aquellos terrenos de extensión reducida y que los campos de grandes dimensiones, al no permitir su fertilización mediante estiércol, requieren del barbecho trabajado. M. Barceló (1995) sugirió, creemos que correctamente, que la opinión de Ibn Bașạāl a este respecto puede interpretarse, tanto como una evidencia de que estercolar era preferido a barbechar como que la capacidad de trasporte de estiércol era limitada, siendo ambas explicaciones posibles y no excluyentes entre sí ${ }^{15}$.Volveremos sobre esta cuestión más adelante. Asimismo, del estudio que J. E. Hernández Bermejo y E. García Sánchez (2008) realizaron sobre las referencias al cultivo de gramíneas que aparecen en los mismos libros de agricultura, se desprende la importancia del uso y aprovechamiento de la paja y las rastrojeras para la ali-

14. Como señala Sigaut (1988: 17), barbechar no significa simplemente dejar descansar la tierra, sino que engloba una serie de labores del terreno agrícola entre primavera y verano necesarias para la siembra de otoño.

15. TRILlo (2004: 60) opina, contrariamente a BARCELó (1995) y siguiendo de cerca los postulados de Watson (inédito), que el comentario de Ibn Ba 1 reflejaría la disociación entre ganadería y agricultura en las explotaciones andalusíes, sobre la base de que una finca amplia podría estercolarse siempre que hubiera una cabaña suficientemente numerosa. 
mentación de los ganados, reflejada en la abundante presencia de términos como zar', aplicado a las gramíneas cultivadas para el empleo de sus frutos y semillas como alimento de ganado (incluidos los cereales-pienso); nașī, referido a pratenses de siega utilizadas como forraje en verde; o halī, estas últimas una vez segadas y secas (henificadas). Por último, en el Libro de Repartimiento de Vélez Málaga se incluye una mención a las quejas manifestadas por los repobladores por el hecho de que los moros metan e traygan sus ganados en ellas [las tierras] al tiempo que aran y cogen los panes, seyendo como diz que es asy costumbre entre los moros e que asy se solía usar e usaba entre ellos (López de Coca, 1977: 362). Estas breves referencias a las que hemos hecho mención creemos que permiten sugerir que el grado de integración entre ganadería y agricultura en al-Ándalus que se refleja en las mismas fuentes documentales empleadas por Watson para sugerir la disociación entre ambas actividades pudo ser mayor del que este reconoció.

En otro orden de cosas, ya hemos apuntado que uno de los elementos de estudio a los que mayor atención se ha dedicado desde el ámbito de la investigación histórica sobre al-Ándalus ha sido el de la intensificación de la producción agrícola. La adopción como parte esencial de la economía de subsistencia de las comunidades rurales andalusíes de la agricultura irrigada y la intensificación de las tareas agrícolas que esta implica ha sido interpretada como una estrategia tendente a la reducción del riesgo productivo (Glick, 2004; Horden \& Purcell, 2000; Retamero, 2008). Aunque volveremos sobre ello más adelante, es necesario señalar que la evidencia arqueozoológica disponible está permitiendo identificar el desarrollo de un proceso similar de intensificación de la producción ganadera durante época andalusí que coincidiría temporalmente con el mayor desarrollo y difusión de la agricultura irrigada.

Discrepamos con F. Retamero (2011: 40) cuando sugiere que la única solución productiva que permite la gestión de una cabaña ganadera, sin que esta actividad se convierta en una especialización de altísimo riesgo, pasa por la inclusió de camps de cereals fora dels perímetres irrigats en el disseny dels espais agrícoles. Gracias al trabajo de T. Glick (1970: 30) sabemos que, a pesar de que las cabañas ganaderas tenían vedada la entrada a la zona de la huerta de Valencia (razón por la cual se destinaron áreas especiales dedicadas a pastos, bovalar, fuera del perímetro irrigado), una vez que el cereal de invierno cultivado en regadío había sido cosechado, el terreno no se sembraba inmediatamente con vegetales, sino que se dejaba en rastrojera que, presumiblemente, sería aprovechada por pequeños rebaños. Así pues, los ritmos agrícolas que se establecen en los campos de cultivo irrigados permiten que el ganado entre a ellos para el aprovechamiento del rastrojo una vez se ha levantado la cosecha, incluso en áreas en las que la presencia del regadío es dominante, tal y como ocurre actualmente en la Vega de Granada (Fig. 1). 


\section{FIGURA 1}

Ejemplo de integración entre agricultura y ganadería en zona de regadío (Vega de Granada). En una misma parcela, tras la cosecha del cultivo irrigado (en este caso habas; arriba), el rastrojo es aprovechado por el ganado (abajo).

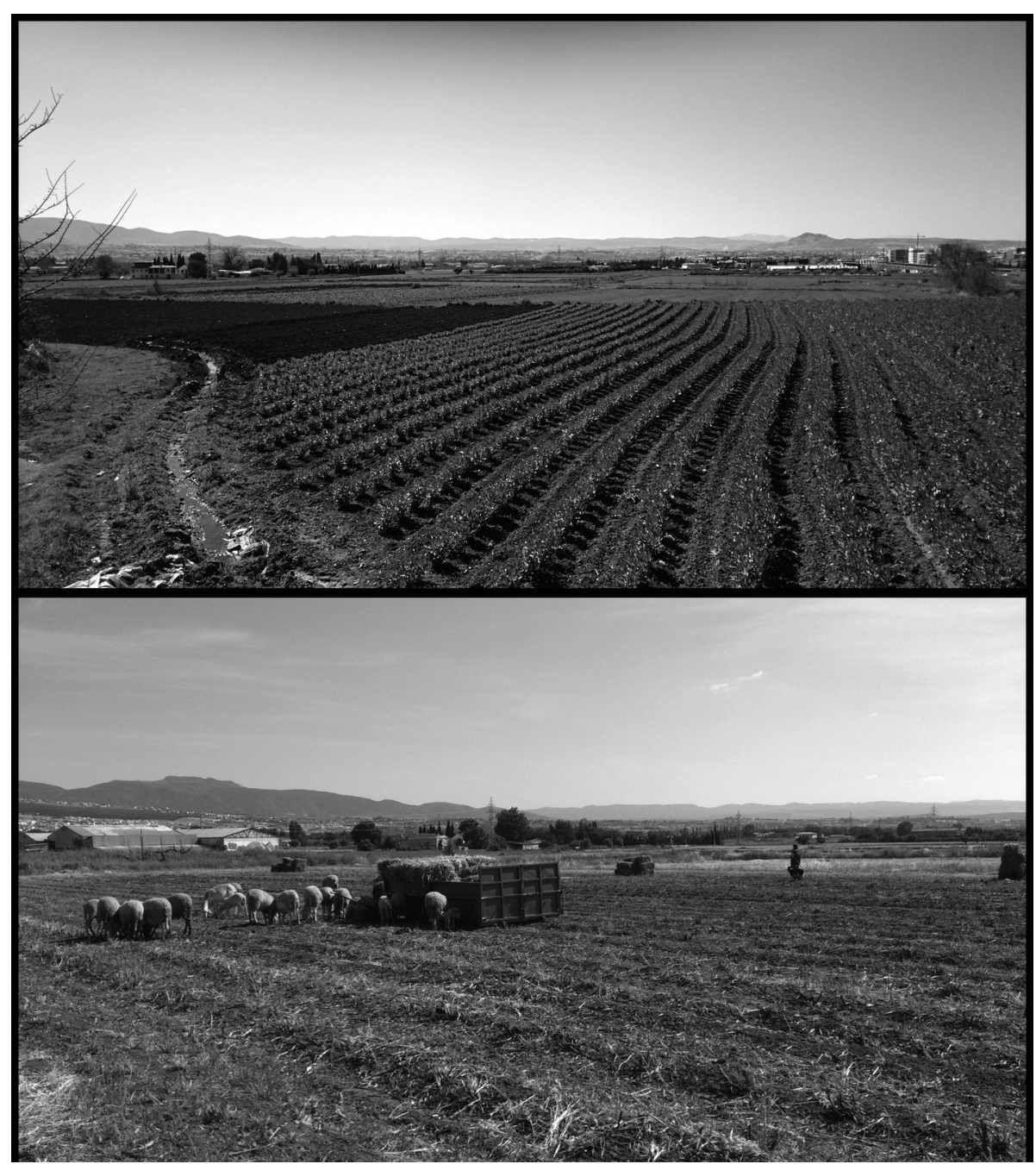

Fuente: imagen de M. García García.

No menos importante nos parece el hecho de que la agricultura de regadío, lejos de suponer un límite a la explotación ganadera, debió de favorecer realmente su desarrollo, gracias a que permitió la producción de cultivos dedicados principalmente a su uso como forraje, pero que podían ser consumidos igualmente, en caso de necesidad, por las poblaciones humanas. En el trabajo al que acabamos de referirnos, T. Glick (1970) constata el cultivo mediante irrigación de alfalfa y algarrobo en la huerta de Valencia, de lo que 
se puede extraer que la alimentación del ganado no dependía exclusivamente de las áreas destinadas a pastos fuera de la zona de huertas. Los tratados de agronomía andalusíes nos informan igualmente sobre el cultivo intensivo de otros dos forrajes verdes regados (kasill), que, además de la alfala (fișfișah), eran segados anualmente y, en ocasiones, dos veces al año como el trébol de Alejandría (kurt) o el guisante gris (naŷîl) (Bolens, 1994: 147-148). Estas fuentes de alimento animal cultivadas en regadío pueden ser suministradas a los animales en pequeñas cantidades debido a que son altamente nutritivas, por lo que su rendimiento como alimento para los rebaños resulta ser extraordinariamente alto (Forbes, 1998). Por tanto, mediante el cultivo de especies propiamente forrajeras o el uso de cereales o leguminosas como la arveja, el haba, la alubia o la lenteja, que pueden emplearse tanto para el consumo humano como animal, la agricultura irrigada permite paliar en cierta medida la falta de pastos naturales durante la época seca, uno de los argumentos centrales propuestos desde la historiografía agraria tradicional sobre los que descansa el modelo clásico de disociación entre el sector agrícola y el ganadero en el Mediterráneo (Barker, 1985; Halstead, 1987, 1996; Hodkinson, 1988; Koster \& Koster, 1976).

Otra cuestión que consideramos de enorme relevancia, al estar estrechamente relacionada con la integración entre agricultura y ganadería en al-Ándalus, es la concerniente al funcionamiento de los procesos físico-biológicos que se encuentran en la base de las prácticas agrícolas intensivas. El trabajo, pionero en este sentido, de L. Bolens (1994) a partir de las referencias contenidas en las fuentes geopónicas árabes permitió corroborar la importancia otorgada por los autores andalusíes al manejo de la fertilidad de la tierra, lo que incide sobre la necesidad de integrar esta cuestión en el análisis del proceso de difusión, gestión y explotación de la agricultura irrigada en al-Ándalus, habida cuenta de que la consideración de los sistemas de fertilización es una herramienta fundamental para entender el crecimiento agrario y los procesos de intensificación en los usos agrícolas del suelo (Garrabou \& González de Molina, 2010; Garrabou, Tello \& Olarieta, 2010).

Es sabido que, con anterioridad al uso de energías fósiles, el potencial productivo de los agroecosistemas mediterráneos estuvo limitado por dos factores esenciales. De un lado, el régimen pluviométrico y la disponibilidad de agua, de los que depende la producción de biomasa debido al papel que esta desempeña en el proceso de fotosíntesis sobre el que se apoya la actividad agraria (Naredo, 1999). De otro lado, la cantidad de nutrientes disponibles para reintroducir en los suelos cultivados con los que compensar los extraídos por las cosechas, un elemento igualmente crucial para evitar la degradación del suelo mediante el mantenimiento equilibrado del balance de nutrientes (Garrabou, Tello \& Olarieta, 2010). La difusión de la agricultura de regadío durante el período andalusí permitió superar el primero de esos constreñimientos, ya que, gracias al aporte hídrico, unido 
a las condiciones de alta temperatura, fue posible la aclimatación de nuevos cultivos y la apertura de una nueva estación agrícola (Watson, $1998^{16}$ ). Sin embargo, en el espacio irrigado, y al contrario de lo que apunta una idea bastante extendida en la literatura sobre el mundo rural andalusí (Barceló, 1995: 244; Bolens, 1994: 194; Glick, 1970: 26; Trillo, 2004: 63), el agua no es la que abona la tierra porque, en sí misma, no es un fertilizante. A pesar de que el agua contiene pequeñas cantidades de nutrientes - sin embargo, insuficientes-, las prácticas de irrigación pueden más bien provocar pérdidas de nutrientes a través de lixiviación (FAO, 1999). Por ello, el carácter intensivo de la agricultura irrigada genera, de hecho, una aceleración de los procesos de degradación de las propiedades del suelo que lo abocan a la insostenibilidad por la caída de la fertilidad, siempre y cuando no se establezcan mecanismos de reposición externa regular de materia orgánica capaz de estabilizar la disponibilidad de nutrientes y la estructura biofísica del suelo para futuras cosechas (Naredo, 1996).

Es, por lo tanto, el estiércol, la materia fertilizadora de toda la vida (Cascón, 1948: 3), el elemento crucial que favorece el aporte esencial de nitrógeno, el elemento nutritivo que habitualmente actúa como factor limitante en la producción agrícola tradicional (Saguer \& Garrabou, 1996). A pesar de que, en la agricultura premecanizada, los nutrientes aportados en forma de estiércol y abono rara vez llegaban a cubrir los extraídos por las cosechas, en realidad, la función principal del estercolado es, siguiendo a Naredo (1996: 23), la de activar la fabricación natural de este nutriente que se opera en los suelos ${ }^{17}$. Es por ello que, en palabras de P. Halstead (2006: 47), to maintain good medium-and long-term levels of crop production in the Mediterranean without long fallow periods, it is necessary to manure fields $[\ldots]^{18}$.

Así pues, la disponibilidad de estiércol es un elemento crítico para el desarrollo en época preindustrial de métodos agrícolas intensivos (Gallant, 1991) en general, y de la

16. Las tesis de Watson no están, tampoco a este respecto, exentas de problemas (e.g., AuBAILE-SALLENAVE, 1984; DECKER, 2009; SQUATRITI, 2014; VAN DER VEEN, 2010; ROWLEY-CONWY, 1989); y es que, como apunta VAN DER VEEN (2010), las innovaciones agrícolas se derivan más de cambios y adaptaciones que de innovaciones y adopciones.

17. Al hilo de esta cuestión, es importante señalar que el abono verde (la incorporación de leguminosas en los ciclos de rotaciones que permitan la fijación de nitrógeno en el suelo), que se ha considerado en ocasiones como sustitutivo del estercolado, no es sostenible a largo plazo ya que, además de consumir una gran cantidad de potasio, provoca severos problemas de patologías debido a la repetición demasiado frecuente de leguminosas en un mismo terreno (GonZÁlez DE MOLINA \& POULIQUEN, 1996).

18. En términos muy similares se expresa CARA (2002: 490) cuando señala que, aunque una adecuada rotación de cultivos favorece la abundancia de las cosechas, no es posible reducir el tiempo de barbecho sin abonar. 
agricultura de regadío en particular, lo que explica, por ejemplo, la prohibición de retirar estiércol de la zona de huerta en torno a Borja a mediados del siglo XIX (García Manrique, 1960: 73) o que, en la huerta de Valencia en el siglo XV, los montones de estiércol fueran objeto de robo ${ }^{19}$, lo que puede indicar tanto el carácter preciado de este «artículo» como su escasez. La importancia del estiércol es, por lo tanto, esencial para el regadío. Lo sabía tanto G. Alonso de Herrera cuando, a principios del siglo XVI, señaló que el arte de estercolar la tierra es lo que permitía a los moros de la Vega de Granada obtener frutos continuamente y sin interrupción, como lo saben los agricultores actuales de la misma Vega de Granada con los que hemos podido conversar.

La aplicación de esta noción elemental de teoría agronómica al conocimiento del sistema agrario de al-Ándalus no resulta, con todo, una novedad. Ya nos hemos referido a la inconsistencia que, para L. Cara (2002: 457), presenta la idea de una intensificación agrícola promovida únicamente mediante la irrigación que deja al margen el problema del mantenimiento de la fertilidad de los campos así cultivados, que no es otro que el del estiércol. En el mismo sentido se expresa J. M. Martín (2007: 317-341) cuando señala que el agua y la rotación de cultivos son del todo insuficientes para fertilizar los suelos agrícolas, por lo que el aporte considerable de materia orgánica y, en consecuencia, la presencia de animales, fue fundamental para el desarrollo de una agricultura intensiva en alÁndalus. Asimismo, C. Trillo (2000: 338) menciona la existencia en el valle de Lecrín (Granada) de parcelas concretas donde, en época nazarí, se conducía a los animales para hacer estiércol, lo que refleja, creemos, la práctica de la concentración de los animales en espacios específicos próximos al núcleo de población (como establos, corrales y majadas), necesaria para que este producto pueda ser recolectado, así como su importancia decisiva especialmente en una zona en la que, como la misma autora reconoce, los cultivos irrigados eran abundantes.

Creemos poder afirmar, por todo lo dicho, que este subproducto ganadero, cuya importancia en los sistemas agrarios históricos y tradicionales está ampliamente documentada (Halstead, 2014; Jones, 2012; Moreno \& Pimenta, 2011), fue determinante para la difusión de la agricultura irrigada en al-Ándalus, por lo que es necesario destacar su relevancia para entender la relación de mutua dependencia que se establece entre agricultura y ganadería en el agroecosistema irrigado.

19. Blumenthal (2009: 100, n. 78), menciona, entre otros casos de enorme interés, el robo por parte de un esclavo llamado Simonet, con la ayuda de un cómplice inidentificado, de más de treinta carregues (unidad que, en este caso, posiblemente se refiera a la carga que un animal puede transportar) de fem (estiércol). 
En definitiva, y a modo de recapitulación de esta sección, son numerosos los argumentos que permiten cuestionar la visión presente en algunos de los trabajos que han centrado su interés sobre el estudio de la ganadería en al-Ándalus caracterizada por (a) la atribución de un papel marginal al sector pecuario en el modelo del complejo agrario andalusí, derivada esencialmente de (b) la supuesta disociación existente entre este y la agricultura irrigada. Si, tal y como se ha mantenido, la organización de los procesos de trabajo campesino en al-Ándalus se asentó fundamentalmente sobre la autonomía de las decisiones productivas por parte de las comunidades rurales y estas, a su vez, tienden principalmente a la reducción del riesgo productivo como elemento crítico que garantiza la reproducción de sus medios básicos de subsistencia, creemos que cabe otorgar a la gestión de los animales domésticos en al-Ándalus un papel más destacado del que tradicionalmente se le ha asignado.

Como se ha tratado de demostrar, el argumento que sostiene la disociación entre agricultura irrigada y ganadería reduce al extremo la complejidad y potencialidad de los sistemas agrarios desarrollados tradicionalmente en las regiones mediterráneas. La organización social y jurídica del espacio de los asentamientos rurales andalusíes, la flexibilidad de las estrategias productivas pecuarias que permiten su adaptación a unos parámetros ecológicos variables (incluida la integración de las prácticas ganaderas en espacios agrícolas, irrigados o no), la posibilidad que ofrece el regadío para aumentar la cantidad y calidad de alimento para el ganado o la necesidad del estiércol para el mantenimiento de la fertilidad de los terrenos explotados intensivamente son algunos de los factores que, en nuestra opinión, permiten definir un sistema agrario en al-Ándalus en el que agricultura y ganadería, lejos de ser dos actividades hostiles entre sí, debieron mantener una relación simbiótica que resultó, además, mutuamente beneficiosa para ambas. Consideramos, por lo tanto, que la ganadería debe entenderse, al igual que se ha postulado para la agricultura irrigada, como una opción social más a considerar dentro del conjunto de estrategias productivas desarrolladas por las comunidades rurales andalusíes.

Resulta preciso, por lo tanto, tratar de definir algo mejor el modelo de explotación pecuaria que se dio en al-Ándalus, para lo cual la integración del conocimiento derivado del registro documental, las observaciones etnográficas sobre el funcionamiento de los sistemas agrarios tradicionales y la evidencia derivada del estudio arqueozoológico pueden sernos de utilidad. 


\section{EVIDENCIA ARQUEOZOOLÓGICA SOBRE LA GESTIÓNY EXPLOTACIÓN GANADERA EN AL-ÁNDALUS}

Como ya se ha señalado, una de las herramientas de estudio que de manera más directa puede aportarnos información acerca de los patrones de aprovechamiento de las cabañas ganaderas en el pasado es la arqueozoología, consistente básicamente en el análisis de los restos animales derivados de contextos arqueológicos con el fin de conocer el tipo de relaciones que se establecieron entre los grupos humanos pretéritos y el mundo animal, así como evaluar el impacto que estas tuvieron a tres niveles fundamentales: en las comunidades humanas, en los propios animales y en el medio ambiente (Moreno, 2013b). A pesar de que en el ámbito peninsular ibérico el reconocimiento del potencial informativo de las muestras bioarqueológicas (botánicas y zoológicas) procedentes de contextos medievales ha sido tardío con respecto al resto de Europa (Morales, 1996), en los últimos años se ha comenzado a poner de manifiesto la capacidad de esta disciplina como recurso operativo y conceptual que, junto al análisis geoarqueológico, permite mejorar nuestro conocimiento sobre las dinámicas políticas y socioeconómicas de las sociedades medievales (Quirós, 2014a), así como sobre las prácticas agrarias desarrolladas (Quirós, 2014b).

Aunque el análisis del registro arqueozoológico permite explorar una amplia variedad de aspectos del pasado ${ }^{20}$, es su capacidad para ofrecer información histórica de entidad sobre los sistemas de gestión ganadera y los modelos de aprovechamiento de los recursos pecuarios lo que nos interesa destacar en esta ocasión. A lo largo de este último apartado se presentarán, de manera necesariamente sintética, algunos de los resultados más destacados obtenidos a partir de la aplicación de esta línea de investigación a contextos andalusíes $^{21}$, con el objetivo fundamental de ofrecer una lectura agroecológica que contribuya a mejorar nuestra comprensión del sistema agrario y del papel que pudo desempeñar en él la actividad pecuaria.

20. Por ejemplo, la identificación de espacios de producción o consumo, el estudio de actividades artesanales, el análisis de relaciones comerciales, la detección de diferencias identitarias (étnicas, sociales, económicas, de edad, género, etc.) entre diferentes grupos o el examen de procesos relacionados con cambios sociales y culturales (O’CONNOR, 2000; REITZ \& WING, 2008; RUSSELL, 2012; SYKES, 2014).

21. La evidencia sobre la que se basa la siguiente discusión ha sido expuesta en varios trabajos de síntesis sobre la información arqueozoológica disponible para el período andalusí, algunos publicados (MORENO, 2013a; MoRENo \& DAVIS, 2001; MORALES et al., 2011) y otros en fase de elaboración (GARCÍA GARCÍA, en preparación). 


\section{FIGURA 2}

Frecuencias relativas de las principales especies domésticas (caprinos [oveja y cabra], vacuno y porcino) en conjuntos arqueofaunísticos de contextos andalusíes ibéricos en los que el número de restos identificados de estas tres especies considerados conjuntamente es superior a 100

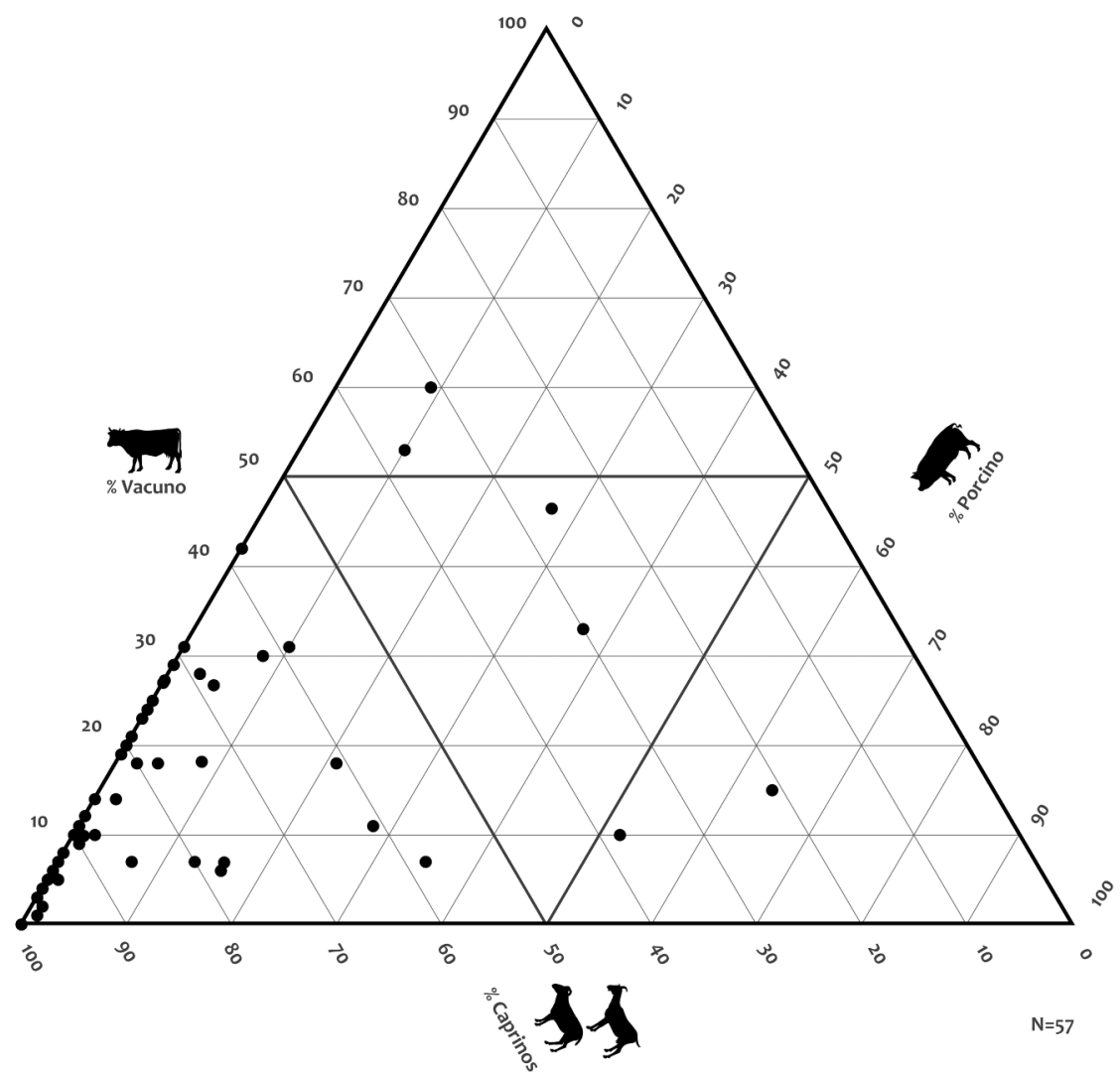

Fuente: elaboración propia a partir de los datos brutos en proceso de elaboración en García García (en preparación).

En primer lugar, debemos comenzar señalando que la mayor parte de los conjuntos arqueozoológicos andalusíes estudiados hasta la fecha proviene de contextos urbanos, y que son escasos -aunque no inexistentes- los análisis de asociaciones arqueofaunísticas procedentes de ámbitos rurales (presumiblemente directamente relacionados con la producción agraria), debido al limitado número de alquerías andalusíes excavadas en extensión en el marco de proyectos de investigación arqueológica (Eiroa, 2012). A pesar de ello, se puede destacar cómo, en la mayor parte de los conjuntos ibéricos (españoles y portugueses) derivados tanto de contextos urbanos como rurales, las frecuencias relativas con 
que aparecen representadas las principales cabañas ganaderas indican una sobrerrepresentación del ganado ovino y caprino en relación con el porcino y al vacuno (Fig. 2$)^{22}$. En aquellos casos en que fue posible analizar en detalle los patrones de abatimiento de ovejas y cabras, los datos coinciden en señalar la abundancia, en términos generales, de cohortes jóvenes, lo que indica una finalidad productiva de los rebaños orientada de forma prioritaria a la producción cárnica, pero que igualmente permitiría el abastecimiento de la demanda doméstica o local de leche, estiércol y, eventualmente, lana.

La evidencia arqueofaunística disponible indica, pues, la preponderancia clara de ovejas y cabras en la economía animal andalusí. Lo que nos interesa destacar en relación a este resultado es el hecho de que, tal y como demuestran numerosos trabajos etnográficos realizados en el ámbito mediterráneo, el modelo de ganadería basado en la explotación a pequeña escala de ovinos y caprinos presenta un elevado nivel de integración potencial con las prácticas agrícolas intensivas. La flexibilidad y versatilidad que caracteriza su explotación ${ }^{23}$ debido a su fácil movilidad, comportamientos etológicos, requerimientos nutricionales y necesidad de agua, favorece la integración de pequeños rebaños de estos animales en sistemas de organización agrícola en los que los espacios irrigados tengan una presencia significativa ${ }^{24}$ (Dahl \& Hjort, 1976; Hodkinson, 1988; Sasson, 2006; Zeder, 1991). Asimismo, se puede señalar que el pastoreo de cultivos inmaduros por ovejas y cabras ha sido tradicionalmente una práctica de gran relevancia en las regiones mediterráneas, empleada para reducir el riesgo de encamado del cereal, un peligro potencial en campos de cultivo altamente fértiles como los irrigados (Halstead, 2006). Por último, pero de no menos relevancia, estos animales aportan un tipo de estiércol altamente apreciado, un subproducto ganadero imprescindible, como hemos visto, para la práctica de una agricultura intensiva.

22. Este resultado coincide en sus líneas generales con lo que sabemos a partir de la información fiscal de que disponemos para época nazarí y morisca, en la que el peso de la ganadería menor es preponderante (RAMOS, 1988; MARTíN, 2007).

23. Lo que S. Hodkinson (1988: 60) denominó el modelo concertina propio de la economía ganadera típicamente mediterránea.

24. El ganado vacuno, en contraposición, necesita mayores cantidades de agua a intervalos más frecuentes, lo que limita la longitud de los movimientos pastorales que pueden realizar diariamente. Además, tiene unos requerimientos nutricionales más selectivos que los de ovejas y cabras ya que precisa de suplementos de forraje durante la mayor parte del año, por lo que su gestión en regiones mediterráneas tradicionalmente se circunscribe principalmente a áreas aluviales o montañosas con buena presencia de agua y, por lo tanto, de biomasa (HALSTEAD, 1995). Cuando su cría se realiza en zonas aluviales o de fondos de valle (por lo tanto, próximas a las áreas de residencia), presenta un mayor nivel potencial de conflicto entre los intereses agrícolas y ganaderos (ZEDER, 1991; DAHL \& HJORT, 1976). 
En segundo lugar, con los datos arqueozoológicos disponibles se puede argüir que, si la baja contribución de restos de porcino corrobora la escasa importancia económica de esta cabaña en al-Ándalus ${ }^{25}$, la limitada presencia de ganado vacuno en el registro arqueozoológico andalusí debe responder a otros factores. Ya que la de vacuno no es una carne especialmente apreciada en los países islámicos (Rodinson, 1965; Rosenberger, 1999), se ha señalado que el valor de este animal en al-Ándalus recaería en su uso como acémila en labores agrícolas (Morales et al., 2011), por lo que su carne solo sería consumida cuando los animales dejaban de ser útiles para el trabajo (García Sánchez, 19831986, 1996). Por nuestra parte, consideramos que la escasa contribución del vacuno en el registro arqueofaunístico puede, además, ponerse en relación con el tipo de sistema agrario imperante en al-Ándalus.

Sobre la base de los costes y beneficios que se derivan del uso del vacuno en labores agrícolas, se ha señalado la relación existente entre sistemas agrarios extensivos (caracterizados por el cultivo de grandes terrazgos con escasos inputs de trabajo) y el uso de fuerza de tracción animal. Al contrario, en sistemas agrarios más intensivos, el cultivo manual de espacios agrícolas de menor escala, altamente productivos gracias a la aplicación de grandes inputs de trabajo humano, hace del uso de acémilas un factor prescindible (Halstead, 1992, 1995; Isaakidou, 2011). De hecho, en aquellos sistemas agrarios en los que los grupos campesinos dirigen su producción esencialmente al consumo doméstico, el mantenimiento de animales de tiro resulta unnecesary and uneconomic (Halstead, 1992: 111), en contraposición evidente al papel crucial que estos animales desempeñan en los sistemas diseñados para la producción de excedentes agrarios a gran escala ${ }^{26}$ (Halstead, 1987, 1996).

Así pues, y aunque resulta necesario tener presente que los restos que componen los conjuntos arqueofaunísticos reflejan patrones de consumo y solo indirectamente patrones de producción (Albarella, 2005; Baker \& Clark, 1993; García García, 2017) y de que se

25. Lo que, invariablemente, refleja el cumplimiento en términos generales de los preceptos islámicos en un ámbito de la existencia humana tan grávido de carga social y cultural como es la alimentación. Existen, sin embargo, excepciones a este respecto. Es el caso, por ejemplo, del arrabal emiral de Cercadilla (Córdoba), donde el examen diacrónico de la frecuencia de restos de suidos (cerdo/jabalí) está permitiendo explorar, desde una óptica de análisis relativamente novedosa como es el estudio de los patrones de consumo alimentario a partir de la evidencia arqueozoológica, el proceso de islamización de una población autóctona entre los siglos VIII y XI (GARCía GARCÍA, 2017, en preparación).

26. Un nivel productivo este que supera el horizonte de subsistencia básico basado en la generación de un excedente previsor (o surplus normal) como precaución contra el riesgo productivo propio de cualquier explotación campesina articulada en torno a la organización autónoma de los procesos de trabajo agrario (HALSTEAD, 1989; HALSTEAD \& O’SHEA, 1989). 
requiere más trabajo en esta dirección ${ }^{27}$, es posible sugerir como hipótesis de trabajo que la evidencia arqueozoológica no solo indica que la carne de vacuno era poco consumida en al-Ándalus, sino que refleja igualmente un uso limitado de la fuerza de tracción de este animal en tareas agrícolas ${ }^{28}$. Este patrón de explotación del vacuno sería, además, divergente al documentado en conjuntos arqueofaunísticos medievales del norte peninsular, en los que la presencia de este animal es significativamente mayor que en los andalusíes (Morales et al., 2011) y el uso de su fuerza de tracción, principalmente en los asentamientos rurales (Grau, 2015), junto con el aprovechamiento cárnico, serían los dos objetivos productivos principales. Será necesario en un futuro desarrollar esta línea de análisis en mayor profundidad ya que supone, en nuestra opinión, un factor esencial de diferenciación entre el sistema agrario feudal y el andalusí y, en consecuencia, entre las formas de organización de los procesos de trabajo campesino y los mecanismos de movilización del excedente rural en ambos órdenes sociales.

En tercer lugar, disponemos de datos arqueozoológicos cada vez más sólidos y abundantes que sugieren que, durante época andalusí, se produjo un proceso de intensificación de la producción ganadera. El análisis biométrico realizado por S. Davis (2008; Davis et al.,2013) sobre un grupo de asociaciones arqueofaunísticas recuperadas de diversos yacimientos del sur de Portugal indica que, durante la fase islámica, el ganado ovino aumentó de tamaño con respecto a época romana. Aunque este patrón ha sido evidenciado por Davis para los siglos XII-XIII, en el caso del conjunto derivado del arrabal cordobés de Cercadilla sobre el que nos encontramos actualmente trabajando, la misma tendencia puede retrotraerse al período califal, apreciándose un aumento evidente en la talla de este animal entre los siglos VIII-IX y el siglo x (Fig. 3) (García García, en preparación).

Debido a que existe una correlación positiva entre el tamaño de los huesos de oveja y el rendimiento cárnico de sus carcasas (Hammond, 1960: 131), esta evidencia puede reflejar, en la línea de lo propuesto por Davis, la especialización en la producción cárnica de este animal orientada al abastecimiento de centros urbanos, reflejando de este modo la preferencia dietética de la sociedad islámica hacia la carne de cordero y borrego de la que nos

27. Por ejemplo, a través del estudio de las osteopatologías sobre restos de bóvidos y équidos, que se ha demostrado como un buen indicador del uso de estos animales en tareas agrícolas y la intensidad del trabajo realizado (BARTosiewicz, NeER \& LeNTACKer, 1997; TELldAhL, 2005).

28. La idea de que, en al-Ándalus, los animales no son tan precisos para la labranza como lo son en las agriculturas mayoritariamente de secano ya fue adelantada, por ejemplo, por MALPICA (2011) sobre la base de los censos de animales que se hicieron tras la conquista castellana en la zona del obispado de Málaga (RAMOs, 1988), donde la incidencia en la tributación de las vacas de arada es significativamente inferior a las de leche. En la misma dirección apuntan los datos recogidos por TRILLO (1998) para la Alpujarra, ya que el peso fiscal del derecho de los pares (que gravaba las yuntas) poco después de la conquista es, en todas las $t \bar{a}$ ' $a$, muy inferior al del ganado mayor y menor. 
informan las fuentes documentales (Arié, 1974-1975; Díaz García, 1985; García Sánchez, 1983-1986, 1996; Martínez Enamorado, 2009). Si el origen de este fenómeno se debió a la mejora zootécnica de las cabañas locales o a la introducción en la Península de variedades o morfotipos alóctonos no es posible discernirlo en el estado actual de la investigación. Sin embargo, lo que sí se puede argüir es que el aumento de talla de la cabaña ovina refleja un patrón de intensificación ${ }^{29}$ de la economía animal durante época andalusí que, a nuestro juicio, debe ser integrado junto al desarrollo de la agricultura de regadío en el mismo paquete de análisis, ya que no parece casual la concurrencia de estrategias de intensificación en los dos principales subsectores agrarios (el agrícola y el ganadero). Resulta necesario, por lo tanto, examinar con mayor detalle la relación que pudo haber existido entre ambos fenómenos y, por extensión, entre ambas prácticas.

\section{FIGURA 3}

\section{Histogramas de las medidas ${ }^{30}$ de los restos poscraneales de oveja recuperados del arrabal de Cercadilla (Córdoba)}
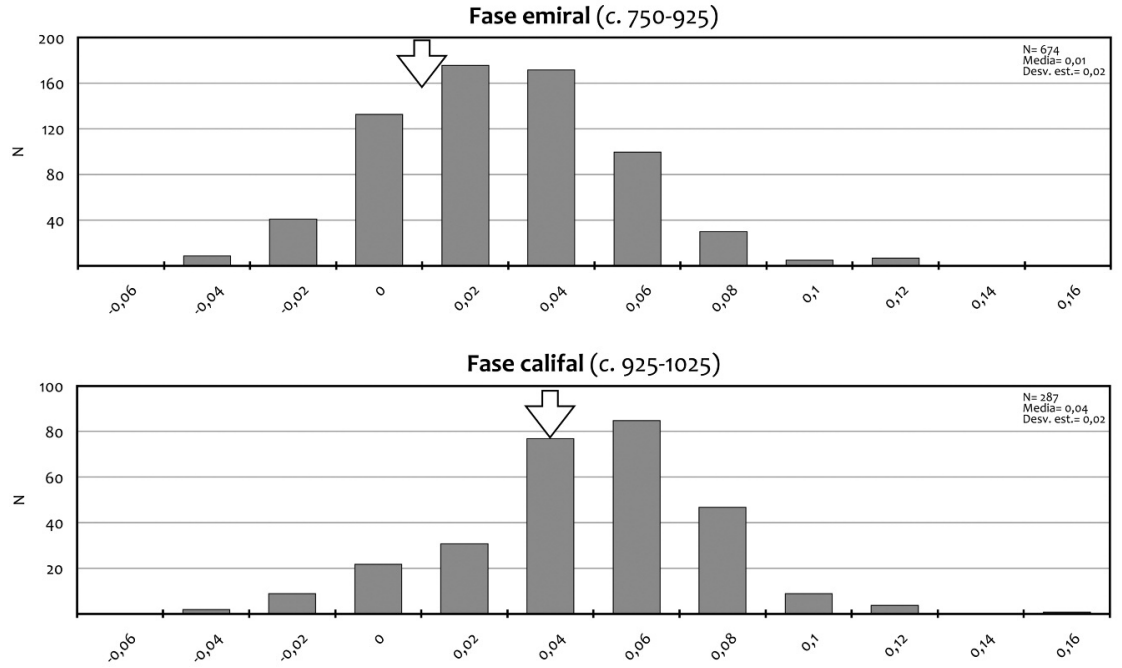

Nota: la media de cada conjunto de datos aparece señalada con una flecha. Se aprecia un aumento de talla entre la fase emiral y la califal.

Fuente: elaboración propia a partir de los datos brutos en proceso de elaboración en García García (en preparación).

29. Entendiendo intensificación ganadera como un tipo de gestión de los animales orientado al aumento de la cantidad de alimento que estos pueden producir (SERJEANTSON, 2007).

30. La técnica empleada para el análisis biométrico en este caso se denomina log ratio (MEADOw, 1999) y permite la comparación de nuestras medidas con un estándar (en este caso, la media de una población de Shetland hembras no mejoradas [DAVIS, 1996]), facilitando así la detección de patrones diacrónicos en relación al tamaño de los animales. Todas las medidas se han combinado en un mismo eje debido al tamaño reducido de las muestras. 
De esta breve síntesis sobre algunos de los resultados más relevantes obtenidos a partir de la aplicación del análisis arqueozoológico en contextos andalusíes se puede extraer una serie de ideas que consideramos de interés. La evidencia arqueozoológica disponible actualmente para el período andalusí permite sugerir, no sin la cautela necesaria, la práctica de un patrón generalizado de explotación ganadera articulado principalmente en torno a la gestión intensiva de pequeños rumiantes domésticos probablemente a pequeña escala, un tipo de economía animal que resulta ser altamente congruente con una dedicación agrícola igualmente intensiva, o que al menos no supondría un obstáculo evidente a la difusión del agroecosistema irrigado, tal y como en ocasiones se ha mantenido. Esta interpretación entronca, por lo tanto, con la línea de argumentación defendida por L. Cara (2002: 491) cuando señala que, en al-Ándalus, más que de agricultura integrada, habría que hablar de ganadería estante funcional, donde ganado y cosechas irrigadas entran en simbiosis, cada una dependiendo y reforzando a la otra. Una vez más, J. M. Martín (2007: 337-340) alcanza conclusiones similares cuando señala que el tipo de explotación ganadera que describen los textos para época nazarí y morisca en el Zenete (al norte de Sierra Nevada), en el que la mayor parte de los agricultores son, a la vez, propietarios de un número pequeño pero suficiente de animales (cabras y ovejas, sobre todo) destinados principalmente a la producción de estiércol para el abonado de los campos, resulta indisociable y necesario para el desarrollo de la agricultura intensiva, por lo que sugiere que deberían haber nacido de manera simultánea.

Esta propuesta, sin embargo, ha de entenderse como una característica general de la economía animal andalusí y no como un rasgo definitorio suyo que lleve a postular la práctica en al-Ándalus de un único sistema de explotación ganadero. De hecho, los datos manejados permiten sugerir la existencia de varios sistemas ganaderos diseñados en función de las necesidades y demandas de la economía local y regional que habrá que estudiar en cada caso. Asimismo, es necesario precisar que la intensificación de la producción cárnica del ovino (inferida a partir del aumento de la talla de este animal) a la que nos hemos referido indica un patrón de optimización productiva de la explotación ganadera dirigido a un mejor aprovechamiento de los recursos animales que, si bien es cierto que favorece la generación de excedente comercializable, no debe entenderse necesariamente como el reflejo del desarrollo de una ganadería comercial de tipo especializado y a gran escala productiva como la que define al pastoralismo propio del mundo feudal ${ }^{31}$. En otras

31. Este tipo de pastoralismo se caracteriza por una enorme escala productiva (al concentrar grandes rebaños, controlados, además, por particulares), un alto nivel de especialización (al estar dedicado prioritariamente al manejo de ovino) y una orientación eminentemente comercial destinada a la producción de lana y cuero, fundamentalmente. Para BARCELó (1988: 209-217) y MARTínez ENAMORADO (2003: 177-8) no existe ninguna evidencia que permita sostener la existencia de un sistema pecuario como este en al-Ándalus. 
palabras, la intensificación de la producción ganadera no es solo posible mediante el aumento de la escala productiva a través de la gestión de grandes rebaños, ya que existen diversas estrategias ganaderas (como la cría selectiva o el mejoramiento de la alimentación animal) que permiten el incremento cuantitativo y cualitativo de la productividad por unidad de producción (individuo animal) sin que sea necesario el desarrollo de un sistema pecuario altamente especializado y de gran escala ${ }^{32}$ (Forbes, 2000; Fratkin \& Smith, 1994; Halstead, 1996; Koster \& Koster, 1976; Makarewicz, 2013). Por ello, resulta importante determinar si el patrón de intensificación de la explotación ganadera que comenzamos a vislumbrar a partir del registro arqueofaunístico para época andalusí responde, bien a una estrategia productiva diseñada de manera "autónoma» por las comunidades rurales ${ }^{33}$, bien al desarrollo de un tipo de pastoralismo más especializado y de mayor escala dirigido al abastecimiento de recursos animales ${ }^{34}$ a los centros urbanos, quizá mantenido y fomentado por el Estado ${ }^{35}$. Creemos que esta es una cuestión de enorme interés sobre la que es posible y necesario continuar trabajando.

\section{CONCLUSIONES}

El objetivo de este trabajo ha sido el de presentar una serie de reflexiones acerca de un tema de investigación al que, salvo estimables excepciones, no se le ha dedicado la atención que creemos merece. Debido, de un lado, al arraigo de una concepción del mundo rural en al-Ándalus definida por la disociación entre agricultura de regadío y ganadería y, de otro, por un enfoque historiográfico definido por L. Cara (2009: 174) como desfasado y estático, el estudio del sector pecuario no ha sido, a nuestro juicio, satisfactoriamente integrado en el análisis del sistema agrario andalusí.Y ello es así porque se ha soslayado un elemento esencial, como se ha tratado de demostrar, en el marco de las

32. Resulta preciso apuntar, al hilo de esta cuestión, que las estrategias de intensificación de la producción agraria no siempre responden a motivaciones de tipo económico, sino que representan una entre varias de las respuestas posibles a un conjunto de condicionantes que pueden ser, también, de naturaleza social, cultural, ideológica o psicológica (VAN DER VEEN, 2010; VAN DER VEEN \& O'CONNOR, 1998).

33. Orientada, quizá, a la producción de excedentes agrarios que puedan ser intercambiados por moneda en el circuito de mercados rurales y urbanos con la que satisfacer la exigencia fiscal.

34. No sólo alimentarios, sino también de materias primas, como lana, piel o hueso necesarios para la producción artesanal.

35. Como, por ejemplo, el que se desarrolló en el Reino de Granada durante los años posteriores a la conquista castellana, donde los concejos, como propietarios de las carnicerías, ejercieron el control sobre los espacios destinados a pastos a escala local a partir de la creación y protección de dehesas de uso exclusivo por parte del ganado destinado al aprovisionamiento urbano (CASTRO, 1996, 2001). 
estrategias productivas desarrolladas por las comunidades campesinas preindustriales, agentes protagonistas del funcionamiento de cualquier economía rural. La relevancia del papel que tradicionalmente ha desempeñado el aprovechamiento de las cabañas ganaderas como recurso alimentario "multiuso", medio de transformación de biomasa no digerible directamente por los humanos, almacenamiento indirecto en tiempos de necesidad o proveedor de fertilizante, es un fenómeno ampliamente documentado en gran parte de los estudios históricos y etnográficos sobre el mundo agrario mediterráneo. El elemento ganadero y el agrícola forman un conjunto interdependiente en la economía rural mediterránea premecanizada, por lo que la competición entre ambos debe ser, como apuntan H. Koster y J. Koster (1976: 275), demostrada y no simplemente asumida.

Un elemento central de esta contribución pasa por reconocer que lo realmente importante no es saber qué plantas se cultivaron o qué animales se criaron en el pasado, sino alcanzar a comprender las razones por las cuales los grupos rurales productores desarrollaron unas opciones económicas y no otras. M. Barceló (1997) lo expresó cabalmente cuando señaló que el conocimiento de los procesos de trabajo campesino es la condición de la inteligibilidad de todo el sistema social. Así pues, al igual que la agricultura irrigada, el aprovechamiento ganadero no es solo una estrategia que permite, a través de mecanismos de intensificación y diversificación a los que se ha aludido, disipar el riesgo productivo, sino que además genera la producción de excedentes cuya gestión (como la de los producidos en los campos irrigados) es necesario estudiar. En consecuencia, las estrategias de manejo y gestión ganadera deben ser consideradas como una opción social y, como tal, han de incorporarse a la discusión de las estrategias agrarias adoptadas por los grupos campesinos andalusíes, lo que permitirá evaluar las medidas de su producción y valorar su dimensión agraria (Kirchner, 2011).

Actualmente disponemos de herramientas de análisis para hacerlo. La arqueozoología es una de ellas, y hemos señalado cómo algunos de los resultados obtenidos a partir de esta línea de investigación, cuando son analizados desde un enfoque agroecológico, reflejan un modelo generalizado de economía animal en al-Ándalus que presenta un buen nivel de integración potencial con un sistema agrario en el que la irrigación sea el factor definitorio. La supuesta contradicción entre agricultura irrigada y ganadería en al-Ándalus que, como también se ha apuntado, es uno de los rasgos que presiden el panorama historiográfico sobre el sector pecuario andalusí, se desvanece, por lo tanto, tan pronto como se cuestiona la validez en términos agroecológicos de dicha noción, se efectúa una lectura alternativa a la generalmente realizada de la documentación escrita o se acude a un tipo de registro arqueológico extraordinariamente informativo como es el arqueozoológico. 
La cuestión, obviamente, no queda cerrada. El recurso a nuevas estrategias de investigación y la integración de informaciones derivadas de otras disciplinas como la arqueobotánica, la geoarqueología, la etnoarqueología o las técnicas biomoleculares resulta esencial, ya que nos permitirá construir un marco de estudio en el que podamos encuadrar nuevas interpretaciones sobre el sistema agrario andalusí desde una perspectiva más orgánica y holística. Es nuestra intención que estas reflexiones sirvan para estimular la investigación en este sentido y demostrar, de paso, la potencialidad de los estudios bioarqueológicos para mejorar nuestro conocimiento sobre las prácticas agrarias en el pasado.

\section{AGRADECIMIENTOS}

Una primera versión de este trabajo fue presentada en el III Seminario Anual de la Sociedad Española de Historia Agraria (SEHA) celebrado en Madrid el 28 de noviembre de 2014. La versión definitiva se ha visto enriquecida por los comentarios y críticas recibidas en el marco de aquella reunión, así como por las opiniones de dos de los evaluadores externos de Historia Agraria.

\section{REFERENCIAS}

Albarella, U. (2005). Meat Production and Consumption in Town and Country. En K. GiLEs \& CH. Dyer (Eds.), Town and Country in the Middle Ages: Contrasts, Contacts and Interconnections, 1100-1500 (pp. 131-148). Leeds: Maney.

Alonso de Herrera, G. (1996 [1513]). Agricultura general. Madrid: Ministerio de Agricultura, Pesca y Alimentación.

Álvarez De Morales, C. (1990). La zootecnia en los textos agrícolas árabes. En E. GARCÍA SÁnchez (Ed.), Ciencias de la Naturaleza en al-Ándalus: Textos y estudios I (pp. 81-91). Granada: Consejo Superior de Investigaciones Científicas.

ARGENTE DEL CASTILLO, C. (1991). La ganadería medieval andaluza, siglos XIII-XVI (reinos de faén y Córdoba). Jaén: Diputación Provincial de Jaén.

ARIÉ, R. (1974-1975). Remarques sur l'alimentation des musulmans d'Espagne au cours du Bas Moyen Age. Cuadernos de Estudios Medievales y Ciencias Técnicas Historiográficas, (2-3), 299-312.

Aubaile-SallenaVe, F. (1984). L'agriculture musulmane aux premiers temps de la conquête: Apports et emprunts; à propos de Agricultural innovation in the early Islamic world de Andrew M. Watson. Fournal d'agriculture traditionnelle et de botanique appliquée, 31 (3-4), 245-256. 
Audoin-Rouzeau, F. (1993). Hommes et animaux en Europe de l'époque antique aux temps modernes. Paris: Centre National de la Recherche Scientifique.

BAKER, P. \& ClaRK, G. (1993). Archaeozoological Evidence for Medieval Italy: A Critical Review of the Present State of Research. Archeologia Medievale, (20), 45-77.

BARCELó, M. (1988). La arqueología extensiva y el estudio de la creación del espacio rural. En M. Barceló, H. Kirchner, J. M. Lluró, R. Martí \& J. M. Torres (Eds.), Arqueología medieval en las afueras del «medievalismo» (pp. 195-274). Barcelona: Crítica.

BARCELÓ, M. (1989). El diseño de espacios irrigados en al-Ándalus: Un enunciado de principios generales. En L. CARA (Coord.), El agua en zonas áridas: Arqueología e historia: Actas del I Coloquio de Historia y Medio Físico (pp. 13-47). Almería: Instituto de Estudios Almerienses.

BARCELó, M. (1992). Quina arqueologia per al-Andalus? En Coloquio Hispano-Italiano de Arqueología Medieval (Granada, 1990) (pp. 243-252). Granada: Patronato de La Alhambra y Generalife.

BARCELó, M. (1995). Saber lo que es un espacio hidráulico y lo que no es o al-Andalus y los feudales. En J. A. González Alcantud \& A. Malpica (Coords.), El Agua: Mitos, ritos y realidades (pp. 240-254). Barcelona: Anthropos.

BARCELó, M. (1997). ¿Por qué los historiadores académicos prefieren hablar de islamización en vez de hablar de campesinos? En D. ARMADA (Coord.), La prospección arqueológica: Segundos encuentros de Arqueología y Patrimonio (Salobreña, 1991) (pp. 134-144). Granada: Ayuntamiento de Salobreña.

Barceló, M., Kirchner, H., Lluró, J. M., Martí, R. \& Torres, J. M. (Eds.) (1988). Arqueología medieval en las afueras del «medievalismo». Barcelona: Crítica.

Barker, G. (1985). Prehistoric Farming in Europe. Cambridge: Cambridge University Press.

Bartosiewicz, L., Neer, W.van \& Lentacker, A. (1997). Draught Cattle:Their Osteological Identification and History. Tervuren: Koninklijk Museum voor Midden-Afrika.

Blumenthal, D. (2009). Enemies and Familiars: Slavery and Mastery in Fifteenth-Century Valencia. Ithaca: Cornell University Press.

Bolens, L. (1994). Agrónomos andaluces de la Edad Media. Granada: Universidad de Granada.

BRAudel, F. (1953). El Mediterráneo y el mundo mediterráneo en la época de Felipe II. México, DF: Fondo de Cultura Económica.

CAMARERo, I. \& CANO, P. (2011). El harīm (servidumbre) en el mundo rural islámico. En J. M. CARABAZA \& L. C. MAKKi (Coords.), El saber en al-Ándalus: Textos y estudios, $V$ (pp. 93-111). Sevilla: Universidad de Sevilla.

CARA, L. \& Rodríguez López, J. M. (1989a). El pastoralismo en al-Andalus: Aproximación arqueológica en el sureste peninsular. Revista de arqueología, (96), 40-48. 
CARA, L. \& RodRÍGUEz López, J. M. (1989b). El ámbito económico del pastoralismo andalusí: Grandes aljibes ganaderos en la provincia de Almería. En L. CARA (Coord.), El agua en zonas áridas: Arqueología e historia: Actas del I Coloquio de Historia y Medio Físico (pp. 631-653). Almería: Instituto de Estudios Almerienses.

CARA, L. (1988). La ganadería hispano-musulmana en la Comarca de los Vélez. Revista Velezana, (7), 5-16.

CARA, L. (1996). «... y mudaban de pastos con sus ganados»: Una aproximación histórica a la ganadería almeriense. En A. SÁNCHEZ PICón (Ed.), Historia y Medio Ambiente en el territorio almeriense (pp. 49-82). Almería: Universidad de Almería.

CARA, L. (2002). La ganadería medieval en el campo de Tabernas y Los Filabres. En C. Trillo (Ed.), Asentamientos rurales y territorio en el mediterráneo medieval (pp. 456498). Granada: Athos-Pérgamos.

CARA, L. (2004). La ganadería de la ciudad de Granada y la Alpujarra en la primera mitad del siglo Xvi. En M. Barrios \& A. GalÁN (Eds.), La historia del Reino de Granada a debate (pp. 179-207). Málaga: Diputación Provincial de Málaga.

CARA, L. (2009). Huellas de pastores: Observando los paisajes ganaderos de los «extremos» granadinos. En A. MALPICA (Coord.), Análisis de los paisajes históricos: De alAndalus a la sociedad feudal (pp. 169-202). Granada: Alhulia.

CAsCón, J. (1948). El estiércol. Madrid: Ministerio de Agricultura. (Hojas Divulgadoras, $12-48 \mathrm{H})$.

CASTRO, T. DE (1996). La alimentación en las crónicas castellanas bajomedievales. Granada: Universidad de Granada.

CASTRO, T. DE (2001). La organización del comercio alimentario en el reino de Granada en la Baja Edad Media: ¿Una deuda con al-Andalus? Anuario de Estudios Medievales, 31 (2), 843-865.

Cubero, J. I. (Ed.) (2003). El libro de agricultura de Al Awan. 2 vols. Sevilla: Consejería de Agricultura, Pesca y Desarrollo Rural de Andalucía.

DAHL, G. \& HJoRT, A. (1976). Having Herds: Pastoral Herd Growth and Household Economy. Stockholm: University of Stockholm.

DAvIS, S. (1996). Measurements of a Group of Adult Female Shetland Sheep Skeletons from a Single flock: A Baseline for Zooarchaeologists. Fournal of Archaeological Science, 23 (4), 593-612.

DAvIS, S. (2008). Zooarchaeological Evidence for Moslem and Christian Improvements of Sheep and Cattle in Portugal. Fournal of Archaeological Science, 35 (4), 9911010.

Davis, S., Svensson, E. M., Albarella, U., Detry, C., Götherström, A., Pires, E. \& GinjA, C. (2013). Evidencia de mejoras de ovino y vacuno durante época andalusí y cristiana en Portugal a partir del análisis zooarqueológico y de ADN antiguo. Debates de Arqueología Medieval, (3), 241-287. 
DeCKeR, M. (2009). Plants and Progress: Rethinking the Islamic Agricultural Revolution. fournal of World History, 20 (2), 187-206.

Díaz de Durana, J. R. \& Fernández de LARREA, J. A. (2002). Economía ganadera y medio ambiente: Guipúzcoa y el Noreste de Navarra en la Baja Edad Media. Historia Agraria, (27), 43-64.

DíAz GarCíA, A. (1985). La alimentación en el reino nazarí a la luz de un tratado sobre alimentos de la época. En Actas de las II fornadas de Cultura Árabe e Islámica (pp. 177-184). Madrid: Instituto Hispano-Árabe de Cultura.

EIrOA, J. A. (2012). Pasado y presente de la arqueología de las alquerías. Imago temporis: Medium Aevum, (6), 386-406.

FAO (1999). Guia para el manejo eficiente de la nutrición de las plantas. Roma: Organización de las Naciones Unidas para la Agricultura y la Alimentación.

FlanNery, K. V. (1969). Origins and Ecological Effects of Early Domestication in Iran and the Near East. En P. J. Ucko \& G. W. Dimbleby (Eds.), The Domestication and Exploitation of Plants and Animals (pp. 73-100). London: Duckworth.

Forbes, H. (1998). European Agriculture Viewed Bottom-Side Upwards: Fodder- and Forage-Provision in a Traditional Greek Community. Environmental Archaeology, (1), 19-34.

Forbes, H. (2000). Landscape Exploitation via Pastoralism: Examining the "Landscape Degradation" versus Sustainable Economy Debate in the Post-Medieval Southern Argolid. En P. Halstead \& C. Frederick (Eds.), Landscape and Land Use in Postglacial Greece (pp. 95-109). Sheffield: Sheffield Academic Press.

FratKIN, E. M. \& SMITH, K. (1994). Labor, Livestock and Land: The Organization of Pastoral Production. En E. M. Fratkin, K. A. Galvin \& E. Abella Roth (Eds.), African Pastoralists Systems: An Integrated Approuch (pp. 99-112). Boulder: L. Rienner.

Gallant, T.W. (1991). Risk and Survival in Ancient Greece: Reconstructing the Rural Domestic Economy. Cambridge: Polity.

García García, M. (2017). El abastecimiento urbano de productos animales en al-Andalus: Reflexiones en torno a su estudio arqueológico. En S. VILLAR \& M. GARCía GARCía (Eds.), Ganadería y arqueología medieval. Salobreña: Alhulia.

García García, M. (2017). Some Remarks on the Provision of Animal Products to Urban Centres in Medieval Islamic Iberia:The Cases of Madinat Ilbirah (Granada) and Cercadilla (Cordova). Quaternary International, (460), 86-96.

GARCÍA GARCÍA, M. (en preparación). Gestión y explotación de los animales en el sureste de la península ibérica durante la Alta Edad Media (siglos VI-XI): Perspectivas históricas y arqueozoológicas. Tesis doctoral inédita. Granada: Universidad de Granada.

García Manrique, E. (1960). Las comarcas de Borja y Tarazona y el Somontano del Moncayo. Zaragoza: Diputación Provincial de Zaragoza. 
García MARTín, P. (2011). La Mesta de Castilla: Historia y cultura pastoril (siglos XIIIXIX). En A. Mattone \& P. F. Simbula (Eds.), La pastorizia mediterranea: Storia e diritto (secoli XI-XX) (pp. 55-70). Roma: Carocci.

GARCÍA SÁNCHEZ, E. (1983-1986). La alimentación en la Andalucía islámica: Estudio histórico y bromatológico. II: Carne, pescado, huevos, leche y productos lácteos. Andalucía Islámica. Textos y Estudios, (4-5), 237-278.

GARCía SÁNCHEZ, E. (1996). La alimentación popular urbana en al-Andalus. Arqueología Medieval, (4), 219-235.

García-Contreras, G. (2011). Production and Use of Salt in al-Andalus: A State of the Art and a Perspective on its study. En J. KLÁPŠTE \& P. SOMMER (Eds.), Food in the $\mathrm{Me}-$ dieval Rural Environment: Processing, Storage, Distribution of Food (pp. 31-43). Turnhout: Brepols.

García-Contreras, G., MalpicA, A. \& Villar, S. (2013). Sal y ganaderia en el reino de Granada (siglos XIII-XV), un proyecto de investigacion sobre dos importantes actividades economicas en epoca nazari. Debates de Arqueología Medieval, (3), 375-390.

GARRABOU, R. \& GonZÁlez DE Molina, M. (Eds.) (2010). La reposición de la fertilidad en los sistemas agrarios tradicionales. Barcelona: Icaria.

Garrabou, R., Tello, E. \& Olarieta, J. R. (2010). La reposición histórica de la fertilidad y el mantenimiento de las capacidades del suelo: Un elemento fundamental de las «buenas prácticas» agrícolas y su sostenibilidad. En R. GARRABOU \& M. GonZÁLEZ DE MOLINA (Eds.), La reposición de la fertilidad en los sistemas agrarios tradicionales (pp. 23-38). Barcelona: Icaria.

Gerbet, M. C. (2002). La ganadería medieval en la península Ibérica. Barcelona: Crítica.

GLICK, T. F. (1970). Irrigation and Society in MedievalValencia. Cambridge: Harvard University Press.

GLICK, T. F. (2004). Sistemes agrícoles islàmics de Xarq al-Andalus. En J. M. SALRACH (Ed.), Història agrària dels Països Catalans. 2: Edat Mitjana (pp. 45-89). Barcelona: Fundació Catalana per a la Recerca.

Gómez Pantoja, J. (Ed.) (2001a). Los rebaños de Gerión: Pastores y trashumancia en Iberia antigua y medieval. Madrid: Casa de Velázquez.

Gómez Pantoja, J. (2001b). Pastio agrestis: Pastoralismo en Hispania romana. En J. Gómez Pantoja (Ed.), Los rebaños de Gerion: Pastores y trashumancia en Iberia antigua $y$ medieval (pp. 177-213). Madrid: Casa de Velázquez.

GonZÁlez de Molina, M. \& Pouliquen,Y. (1996). De la agricultura orgánica tradicional a la agricultura industrial: ¿Una necesidad ecológica? Santa Fe, 1750-1904. En R. GARRABOU \& J. M. NAREDO (Eds.), La fertilización en los sistemas agrarios: Una perspectiva histórica (pp. 127-169). Madrid: Fundación Argentaria. 
Grau, I. (2015). The Zooarchaeology of Medieval Alava in its Iberian Context. Oxford: British Archeological Reports.

GutiÉRrez LlORET, S. (1995). El origen de la huerta de Orihuela entre los siglos VII y XI: Una propuesta arqueológica sobre la explotación de las zonas húmedas del Bajo Segura. Arbor, (593), 65-94.

HALPERIN, T. (1956). Recouvrement de civilisation: Les Morisques du Royaume de Valence au XVI siècle. Annales. Économies, Sociétés, Civilisations, 11 (2), 154-182.

Halstead, P. \& O’Shea, J. (1989). BadYears Economics: Cultural Responses to Risk and Uncertainty. Cambridge: Cambridge University Press.

HalsteAd, P. (1987). Traditional and Ancient Rural Economy in Mediterranean Europe: plus ça change? Fournal of Hellenic Studies, (107), 77-87.

HALsTEAD, P. (1989). The Economy has a Normal Surplus: Economic Stability and Social Change among Early Farming Communities of Thessaly, Greece. En P. HALSTEAD \& J. O’SHEA (Eds.), Bad Year Economics: Cultural Responses to Risk and Uncertainty (pp. 68-80). Cambridge: Cambridge University Press.

Halstead, P. (1992). From Reciprocity to Redistribution: Modelling the Exchange of Livestock in Neolithic Greece. Anthropozoologica, (16), 19-30.

Halstead, P. (1993). Banking on Livestock: Indirect Storage in Greek Agriculture. Bulletin on Sumerian Agriculture, (7), 63-75.

Halstead, P. (1995). Plough and Power:The Economic and Social Significance of Cultivation with the Ox-Drawn Ard in the Mediterranean. Bulletin on Sumerian Agriculture, (8), 11-22.

Halstead, P. (1996). Pastoralism or Household Herding? Problems of Scale and Specialization in Early Greek Animal Husbandry. World Archaeology, (28), 20-42.

HALsTEAD, P. (2006). Sheep in the Garden: The Integration of Crop and Livestock Husbandry in Early Farming Regimes of Greece and Southern Europe. En D. SERJEANTSON \& D. FiEld (Eds.), Animals in the Neolithic of Britain and Europe (pp. 4255). Oxford: Oxbow.

Halstead, P. (2014). Two Oxen ahead: Pre-Mechanized Farming in the Mediterranean. Chichester: Wiley-Blackwell.

Hammond, J. (1960). Farm Animals: Their Breeding, Growth and Inheritance. London: Edward Arnold.

Hernández Bermejo, J. E. \& García SÁnchez, E. (2008). Las gramíneas en al-Andalus. En E. García SÁnchez \& C. Álvarez de Morales (Eds.), Ciencias de la Naturaleza en al-Andalus: Textos y estudios VIII (pp. 235-288). Madrid: Consejo Superior de Investigaciones Científicas.

Hodkinson, S. (1988). Animal Husbandry in the Greek Polis. En C. R. WhitTaKeR (Ed.), Pastoral Economies in Classical Antiquity (pp. 35-74). Cambridge:The Cambridge Philological Society. 
Horden, P. \& Purcell, N. (2000). The Corrupting Sea:A Study of Mediterranean History. Oxford: Blackwell.

IBN BAșșāL (1995). Libro de agricultura [Trads. y eds.: E. García Sánchez \& J. E. Hernández Bermejo]. Sevilla: Sevilla Equipo 28.

IBN LUYUN (1988). Tratado de agricultura. [Trad. y ed.: J. Eguaras]. Granada: Patronato de la Alhambra y Generalife.

IsAAKIDOU, V. (2011). Farming Regimes in Neolithic Europe: Gardening with Cows and other Models. En A. Hadjikoumis, E. Robinson \& S. Viner (Eds.), The Dynamics of Neolithisation in Europe: Studies in Honour of Andrew Sherratt (pp. 90-112). Oxford: Oxbow.

JONES, G. (2005). Garden Cultivation of Staple Crops and its Implications for Settlement Location and Continuity. World Archaeology, 37 (2), 164-176.

Jones, R. (Ed.) (2012). Manure Matters: Historical, Archaeological and Ethnographic Perspectives. Farnham: Ashgate.

KIRCHNER, H. \& NAVARRO, C. (1994). Objetivos, métodos y práctica de la arqueología hidráulica. Arqueología y territorio medieval, (1), 159-182.

KIRCHNER, H. (2011). Archaeology of the Landscape and Archaeology of Farmed Areas in the Medieval Hispanic Societies. Imago temporis. Medium Aevum, (5), 55-86.

KLeIN, J. (1920). The Mesta: A Study in Spanish Economic History, 1273-1836. Cambridge: Harvard University Press.

Koster, H. A. \& Koster, J. B. (1976). Competition or Symbiosis?: Pastoral Adaptive Strategies in the Southern Argolid, Greece. Annals of the New York Academic of Sciences, (268), 275-285.

KRON, G. (2008). Animal Husbandry, Hunting, Fishing, and Fish Production. En J. P. Oleson (Ed.), The Oxford Handbook of Engineering and Technology in the Classical World (pp. 175-222). Oxford: Oxford University Press.

Lagardère, V. (1993). Campagnes et paysans d'Al-Andalus, VIII $-X V^{e}$ siècles. Paris: Maisonneuve et Larose.

LAGARDĖRE, V. (1996). Terres communes et droits d'usage en al-Andalus ( $\mathrm{X}^{\mathrm{e}}-\mathrm{XV}$ e siècles). Revue du monde musulman et de la Méditerranée, (79-80), 43-54.

LINANT DE BELLEFONDS, Y. (1959). Un problème de sociologie juridique: Les terres "communes" en pays d'Islam. Studia Islamica, (10), 111-136.

LóPEZ DE CoCA, J. E. (1977). El Repartimiento de Vélez Málaga. Cuadernos de Historia, Anexos de Hispania, (7), 357-439.

MaKarewicz, C. A. (2013). A Pastoralist Manifesto: Breaking Stereotypes and Re-Conceptualizing Pastoralism in the Near Eastern Neolithic. Levant, 45 (2), 159-174.

Malpica, A. (2000). El poblamiento y la organización del espacio. En R. G. PeInAdo (Coord.), Historia del reino de Granada. 1: De los orígenes a la época mudéjar (hasta 
1502) (pp. 249-290). Granada: Universidad de Granada/Fundación El legado andalusí.

MalpiCA, A. (2008). Techniques et aménagements des salines médiévales de l'intérieur des terres en Andalousie orientale. En O. Weller, A. Dufraisse \& P. Pétrequin (Eds.), Sel, eau et forêt: D'hier à aujourd'hui (pp. 433-452). Besançon: Presses universitaires de Franche-Comté.

MALPICA, A. (2009). Castillos, alquerías y ciudades en al-Andalus: Un debate partiendo del análisis arqueológico. En A. L. Molina \& J. A. EIrOA (Eds.), El castillo medieval en tiempos de Alfonso X el Sabio (pp. 99-120.) Murcia: Universidad de Murcia.

MALPICA, A. (2011). Poblamiento, agricultura y ganadería en el reino nazarí de Granada. En A. Mattone \& P. F. Simbula (Eds.), La pastorizia mediterranea: Storia e diritto (secoli XI-XX) (pp. 41-54). Roma: Carocci.

MALPICA, A. (2012). La vida agrícola y la ganadería en al-Andalus y en el reino nazarí de Granada. En R. MARín (Ed.), Homenaje al profesor Dr. D. Fosé Ignacio Fernández deViana y Vieites (pp. 213-228). Granada: Universidad de Granada.

MalpicA, A. (Ed.) (2017). Zonas húmedas en Andalucía medieval: Inicio de un debate. Salobreña: Alhulia.

Malpica, A., Villar, S., García, M. \& García Contreras, G. (en prensa). Animal Husbandry and Saltworks in the Kingdom of Granada (13th-15th Centuries): The Dynamics of Landscapes in a Mediterranean Territory. En Mediterranean Landscapes in Post Antiquity: New Frontiers and New Perspectives. Cambridge: Cambridge Scholars Publishing.

MANZANO, E. (2012). Al-Andalus: Un balance crítico. En P. SÉNAC (Ed.), Histoire et archéologie de l'occident musulman (VII-XV'): al-Andalus, Maghreb, Sicile (pp. 19-31). Toulouse: CNRS/Université de Toulouse.

MArston, J. M. (2011). Archaeological Markers of Agricultural Risk Management. fournal of Anthropological Archaeology, 30 (2), 190-205.

Martín, J. M. (2007). Poblamiento y territorio medieval en el Zenete (Granada). Granada: Universidad de Granada.

Martínez Enamorado, V. (2003). Al-Andalus desde la periferia: La formación de una sociedad musulmana en tierras malagueñas (siglos VIII-X). Málaga: Diputación Provincial de Málaga.

Martínez Enamorado, V. (2009). Paladares de príncipes, recetas cortesanas, comidas de campesinos: Valoraciones en torno a la alimentación de los andalusíes. En J. M. Hita, J. SuÁrez Padilla \& F. Villada (Eds.), Comer en Ceuta en el siglo XIV: La alimentación durante la época mariní (pp. 61-98). Ceuta: Ciudad Autónoma de Ceuta.

MEAdow, R. H. (1999). The Use of Size Index Scaling Techniques for Research on Archaeozoological Collections from the Middle East. En C. Becker, H. MANHART, J. PETERS \& J. SCHIBLER (Eds.), Historia Animalium ex Ossibus: Beiträge zur Paläoanato- 
mie, Archäologie, Ägyptologie, Ethnologie und Geschichte der Tiermedizin (pp. 285-300). Rahden: Marie Leidorf.

Montserrat, P. \& Fillat, F. (1990). The Systems of Grassland Management in Spain. En A. I. BreymeYeR (Ed.), Managed Grasslands: Regional Studies (pp. 37-70). Amsterdam: Elsevier.

Morales, A. (1996). A Plea for Recognition: Faunal Analysis as a Historical Discipline. Arqueologia Medieval, (4), 255-265.

Morales, A., Moreno, M., Roselló, E., Llorente, L. \& Morales, D. C. (2011). 711

AD: ¿El origen de una disyunción alimentaria? Zona Arqueológica, 15 (2), 303-322.

Moreno, M. \& DAvis, S. (2001). Estudio de las asociaciones faunísticas recuperadas en Alcácer do Sal, Convento de São Francisco, Santarém y Sé de Lisboa. En Garb, sítios islãmicos do sul peninsular (pp. 231-255). Lisboa/Badajoz: Ministério da Cultura/Junta de Extremadura.

Moreno, M. \& Pimenta, C. (2011). Animal Dung: Rich Ethnographic Records, Poor Archaeozoological Evidence. En U. Albarella \& A. Trentacoste (Eds.), Ethnozooarchaeology: The Present and Past of Human-Animal Relationships (pp. 20-28). Oxford: Oxbow.

Moreno, M. (2013a). Gestión y aprovechamiento de cabañas ganaderas en al-Andalus: Aportaciones desde la arqueozoología. Debates de Arqueología Medieval, (3), 75-98. Moreno, M. (2013b). Arqueozoología. En M. García Díez \& L. Zapata (Eds.), Métodos y técnicas de análisis y estudio en arqueología prehistórica: De lo técnico a la reconstrucción de los grupos humanos (pp. 346-366). Bilbao: Universidad del País Vasco.

MuÑoz, A. \& DíAz LóPEz, J. P. (Eds.) (2002). Herbajes, trashumantes y estantes: La ganadería en la península ibérica (épocas medieval y moderna). Almería: Instituto de Estudios Almerienses.

NAREDO, J. M. (1996). Sobre la reposición natural y artificial de agua y de nutrientes en los sistemas agrarios y las dificultades que comporta su medición y seguimiento. En R. GARRABOU \& J. M. NAREDO (Eds.), La fertilización en los sistemas agrarios: Una perspectiva histórica (pp. 17-33). Madrid: Fundación Argentaria.

NAREDO, J. M. (1999). Consideraciones económicas sobre el papel del agua en los sistemas agrarios. En R. GARRABOU \& J. M. NAREDO (Eds.), El agua en los sistemas agrarios: Una perspectiva histórica (pp. 63-76). Madrid: Fundación Argentaria.

O'Connor, T. (2000). The Archaeology of Animal Bones. Stroud: Sutton.

O'Connor, T. (2014). Livestock and Animal Husbandry in Early Medieval England. Quaternary International, (346), 109-118.

OCAÑA, M. DEL C. (1974). La Vega de Granada: Estudio geografico. Granada: Instituto de Geografia Aplicada del Patronato «Alonso de Herrera»/Caja de Ahorros de Granada. 
PAscua, E. (2007). Las otras comunidades: Pastores y ganaderos en la Castilla medieval. En A. Rodríguez (Ed.), El lugar del campesino (pp. 205-233).Valencia: Universidad de Valencia.

Pascua, E. (2011). Communities and Sustainability in Medieval and Early Modern Aragon, 1200-1600. International fournal of the Commons, 5 (2), 535-556.

Quirós, J. A. (2014a). Archeobiologie e archeologia medievale: Dall'archeometria all'archeologia ambientale. Archeologia Medievale, núm. extra (41) 51-62.

Quirós, J. A. (Ed.) (2014b). Agrarian Archaeology in Early Medieval Europe. Quaternary International (346), 1-162.

Ramos, J. R. (1988). Politica ganadera de los Reyes Catolicos en el obispado de Malaga. Málaga: Diputación Provincial de Málaga.

ReItZ, E. J. \& WING, E. S. (2008). Zooarchaeology. Cambridge: Cambridge University Press.

RETAMERO, F. (1998). Un conjunto de reglas sabias y ordenadas: La disciplina agraria del sulțán. En C. Laliena \& J. F. UTrilla (Eds.), De Toledo a Huesca: Sociedades medievales en transición a finales del siglo XI, 1080-110 (pp. 75-91). Zaragoza: Institución Fernando el Católico/Diputación de Zaragoza.

RETAMERO, F. (2002). Gestiones campesinas de llanos y barrancos en el término de Alaior (Menorca): Siglos X-XIII. En C. TRILlo (Ed.), Asentamientos rurales y territorio en el Mediterráneo medieval (pp. 187-220). Granada: Athos-Pérgamos.

Retamero, F. (2008). Irrigated Agriculture, Risk and Population: The Andalusi Hydraulic Systems of the Balearic Islands as a Case Study (10th-13th C). En R. COMPATANGELO (Ed.), Marqueurs des paysages et systèmes socio-économiques: Actes du colloque COST du Mans (7-9 decembre 2006) (pp. 135-148). Rennes: Presses universitaires de Rennes.

Retamero, F. (2011). Pautes per a l'estudi dels conreus de secà a al-Andalus. En F. SABatè (Ed.), Arqueologia Medieval: Els espais de secà (pp. 31-51). Lleida: Pagès.

Rodinson, M. (1965). Ghidha. En C. Pellat \& J. Schacht (Eds.), Encyclopedia of Islam. Vol. 2 (pp. 1057-1072). Leiden: Brill.

Rosenberger, B. (1999). Arab Cuisine and its Contribution to European Culture. En J. L. Flandrin \& M. Montanari (Eds.), Food:A Culinary History from Antiquity to the Present (pp. 207-223). New York: Columbia University Press.

Rowley-Conwy, P. (1989). Nubia AD 0-550 and the "Islamic" Agricultural Revolution: Preliminary Botanical Evidence from Qasr Ibrim, Egyptian Nubia. Archeologie du Nil Moyen, (3), 131-138.

Russell, N. (2012). Social Zooarchaeology: Humans and Animals in Prehistory. Cambridge: Cambridge University Press.

SAGUER, E. \& GARRABOU, R. (1996). Métodos de fertilización en la agricultura catalana durante la segunda mitad del siglo XIX: Una aproximación a los procesos físicos de re- 
posición de la fertilidad de la tierra. En R. GARRABOU \& J. M. NAREDO (Eds.), La fertilización en los sistemas agrarios: Una perspectiva histórica (pp. 89-126). Madrid: Fundación Argentaria.

SALVADORI, F. (2015). Uomini e animali nel Medioevo: Ricerche archeozoologiche in Italia, tra analisi di laboratorio e censimento dell'edito. Saarbrucken: Edizioni Accademiche Italiane.

SAsson, A. (2006). Animal Husbandry and Diet in Pre-Modern Villages in Mandatory Palestine, according to Ethnographic Data. En M. MALTBy (Ed.), Integrating Zooarchaeology: Proceedings of the 9th ICAZ Conference (pp. 33-40). Oxford: Oxbow.

SERJEANTSON, D. (2007). Intensification of Animal Husbandry in the Late Bronze Age? The Contribution of Sheep and Pigs. En C. Haselgrove \& R. Pope (Eds.), The Earlier Iron Age in Britain and the Near Continent (pp. 80-93). Oxford: Oxbow.

Sherratt, A. (1981). Plough and Pastoralism: Aspects of the Secondary Products Revolution. En I. Hodder (Ed.), Pattern of the Past (pp. 261-305). Cambridge: Cambridge University Press.

SigaUt, F. (1988). L'évolution technique des agricultures européennes avant l'époque industrielle. Revue archéologique du Centre de la France, 27 (1), 7-41.

SQuatriti, P. (2014). Of Seeds, Seasons, and Seas: Andrew Watson's Medieval Agrarian Revolution Forty Years Later. The fournal of Economic History, 74 (4), 1205-1220. Sykes, N. (2014). Beastly Questions: Animal Answers to Archaeological Issues. London: Bloomsbury.

Telldahl, Y. (2005). Can Palaeopathology be Used as Evidence for Draught Animals? En J. Davies, M. Fabis, I. Mainland, M. Richards \& R. Thomas (Eds.), Diet and Health in Past Animal Populations: Current Research and Future Directions: Proceedings of the 9th Conference of the ICAZ, Durham August 2002 (pp. 63-67). Oxford: Oxbow.

Torres, J. M. (1988). La zooarqueología. En M. BARCELó, H. KirChNER, J. M. LluRó, R. MARTí \& J. M. Torres (Eds.), Arqueología medieval: En las afueras del «medievalismo» (pp. 134-164). Barcelona: Crítica.

Trillo, C. (1998). La Alpujarra antes y después de la conquista castellana. Granada: Universidad de Granada.

TRILlo, C. (1999). El paisaje vegetal en la Granada islámica y sus transformaciones tras la conquista castellana. Historia Agraria, (17), 131-152.

TRILlo, C. (2000). Las actividades económicas y las estructuras sociales. En R. G. PEINADO (Ed.), Historia del reino de Granada. 1: De los origenes a la época mudéjar (hasta 1502) (pp. 291-347). Granada: Universidad de Granada/Fundación El legado andalusí.

Trillo, C. (2004). Agua, tierra y hombres en al-Andalus: La dimensión agrícola del mundo nazarí. Granada: Ajbar. 
TRILlo, C. (2011). La ganadería en el reino de Granada:Transformación de una actividad económica del dominio islámico al cristiano. En A. Mattone \& P. F. Simbula (Eds.), La pastorizia mediterranea: Storia e diritto (secoli XI-XX) (pp. 629-643). Roma: Carocci.

VeEn, M. van DER \& O’ConNor, T. (1998). The Expansion of Agricultural Production in Late Iron Age and Roman Britain. En J. BAyLey (Ed.), Science in Archaeology (pp. 127-143). London: English Heritage.

VEEN, M. vAN DER (2010). Consumption, Trade and Innovation: Exploring the Botanical Remains from the Roman and Islamic Ports at Quseir al-Qadim, Egypt. Frankfurt am Main: Africa Magna.

VilLAR, S. \& GARCÍA GARCÍA, M. (Eds.) (2017). Ganadería y arqueología medieval. Salobreña: Alhulia.

VILLAR, S. (2016). El poblamiento andalusi del territorio granadino del Quempe. Tesis doctoral inédita. Granada: Universidad de Granada.

Watson, A. M. (1974). The Arab Agricultural Revolution and its Diffusion, 700-1100. The Fournal of Economic History, 34 (1), 8-35.

Watson, A. M. (1981). A Medieval Green Revolution. En A. L. Udovitch (Ed.), The Islamic Middle East, 700-1900 (pp. 29-58). Princeton: Darwin.

Watson, A. M. (1998). Innovaciones en la agricultura en los primeros tiempos del mundo islámico. [Trad.: A. Martínez]. Granada: Universidad de Granada.

Watson, A. M. (2007). A Case of Non-Diffusion: The Non-Adoption by Muslim Spain of the Open-Field System of Christian Europe: Causes and Consequences. En S. CAVACIOCCHI (Ed.), Relazioni economiche tra Europa e mondo islamico, secc. XIII-XVIII: Atti della "XXXVIII Settimana di Studi", Fondazione Istituto Internazionale di Storia Economica "F. Datini”" (pp. 242-265). Firenze: Le Monnier.

Watson, A. M. (inédito). Livestock in the Agriculture of Muslim Spain:Was Farming "Integrated"? And Why does it Matter? En De la sociedad islámica a la feudal:Veinte años de Al-Andalus: Homenaje a Pierre Guichard. Granada-València, 9-14 de mayo.

WickHAm, C. (1983). Pastoralism and Underdevelopment in the Early Middle Age. Settimane di Study del Centro Italiano di Studi sull'Alto Medioevo, (31), 401-455.

Zeder, M. A. (1991). Feeding Cities: Specialized Animal Economy in the Ancient Near East.Washington: Smithsonian Institution Press. 